Article

\title{
Simultaneous Wireless Information and Power Transfer for MIMO Interference Channel Networks Based on Interference Alignment
}

\author{
Anming Dong ${ }^{1,3}$, Haixia Zhang ${ }^{2, *}$, Minglei Shu ${ }^{3}$ and Dongfeng Yuan ${ }^{2}$ \\ 1 School of Information, Qilu University of Technology, Jinan 250353, China; anmingdong@qlu.edu.cn or \\ donganming@gmail.com \\ 2 Shandong Provincial Key Laboratory of Wireless Communication Technologies, Shandong University, \\ Jinan 250100, China; dfyuan@sdu.edu.cn \\ 3 Shandong Computer Science Center (National Supercomputer Center in Jinan), Shandong Provincial Key \\ Laboratory of Computer Networks, Jinan 250014, China; shuml@sdas.org \\ * Correspondence: haixia.zhang@sdu.edu.cn; Tel.: +86-531-8836-2525 (ext. 888)
}

Received: 1 July 2017; Accepted: 8 September 2017; Published: 13 September 2017

\begin{abstract}
This paper considers power splitting (PS)-based simultaneous wireless information and power transfer (SWIPT) for multiple-input multiple-output (MIMO) interference channel networks where multiple transceiver pairs share the same frequency spectrum. As the PS model is adopted, an individual receiver splits the received signal into two parts for information decoding (ID) and energy harvesting (EH), respectively. Aiming to minimize the total transmit power, transmit precoders, receive filters and PS ratios are jointly designed under a predefined signal-to-interference-plus-noise ratio (SINR) and EH constraints. The formulated joint transceiver design and power splitting problem is non-convex and thus difficult to solve directly. In order to effectively obtain its solution, the feasibility conditions of the formulated non-convex problem are first analyzed. Based on the analysis, an iterative algorithm is proposed by alternatively optimizing the transmitters together with the power splitting factors and the receivers based on semidefinite programming (SDP) relaxation. Moreover, considering the prohibitive computational cost of the SDP for practical applications, a low-complexity suboptimal scheme is proposed by separately designing interference-suppressing transceivers based on interference alignment (IA) and optimizing the transmit power allocation together with splitting factors. The transmit power allocation and receive power splitting problem is then recast as a convex optimization problem and solved efficiently. To further reduce the computational complexity, a low-complexity scheme is proposed by calculating the transmit power allocation and receive PS ratios in closed-form. Simulation results show the effectiveness of the proposed schemes in achieving SWIPT for MIMO interference channel (IC) networks.
\end{abstract}

Keywords: energy harvesting; interference alignment; interference channel; multi-input multi-output (MIMO); simultaneous wireless information and power transfer (SWIPT); transceiver design; power splitting

\section{Introduction}

Harvesting energy from RF signals is a promising solution to provide perpetual and cost-effective power supplies for wireless networks [1]. Compared with traditional energy harvesting (EH) methods that are dependent on external sources, such as solar power and wind energy, harvesting energy from RF signals makes it possible to supply energy for the wireless devices on demand at any time and thus brings great convenience and guarantees quality of service for wireless systems. Moreover, 
along with the wide adoption of massive multiple-input multiple-output (MIMO) techniques and the shrinking coverage of base stations in the fifth-generation (5G) systems [2-4], it becomes possible to transmit wireless power with higher efficiency in the future. By combing EH with traditional information transmission, it is expected that the power line connected to the mobile devices would be dismissedcompletely in future wireless communications [5]. Due to these demands and technical advances, simultaneous wireless information and power transfer (SWIPT) constitutes one of the main techniques for 5G wireless networks [6], and has aroused wide attention from both academia and industry recently.

The pioneer studies of SWIPT in $[7,8]$ focused on characterizing the fundamental rate and energy trade-off under the assumption that the same signal can simultaneously convey information and power. However, limited by current circuit techniques, it is impossible to achieve simultaneous transfer of information and power with the same signal in practice [9]. Therefore, achieving SWIPT now mainly relies on the architectures of transceivers and operation protocols. In [10,11], time switching (TS) schemes were proposed to realize SWIPT by alternatively decoding information and harvesting energy in a time division manner. The work in [11] also proposed a power splitting (PS) scheme, where the received signals are divided into two parts for information retrieval and energy storage, respectively and simultaneously, at the information decoder and energy harvester. Different from the above schemes, which assume that the EH receiver and ID receiver are co-located and experience the same channel, the SWIPT schemes for separated EH and ID receivers, which experience different channels, can be found in [11-14]. In [11], an MIMO broadcast channel (BC) SWIPT system, which consists of a separated $\mathrm{EH}$ receiver and information receiver, was first studied, and its rate-energy tradeoffs were characterized. For the similar BC SWIPT model with single-antenna receivers, [12] studied beamforming optimization schemes to maximize the weighted sum energy of the $\mathrm{EH}$ receivers subject to given signal-to-interference-and-noise ratio (SINR) constraints at the ID receivers. The design schemes were formulated as nonconvex quadratically-constrained quadratic programs (QCQP) and solved with the help of semidefinite programming (SDP) relaxation (SDR). In [13], the separated MIMO BC SWIPT system with multiple-antenna receivers was studied, and the transceiver designs based on mean-square error (MSE) criteria were proposed under source transmit power and harvested energy constraints. The work in [14] investigated transceiver design schemes for an MIMO BC system with one EH receiver and one ID receiver based on the weighted minimum mean-square error (WMMSE) criterion. As for the PS-based SWIPT systems, following [11], work on joint beamformer/receiver designing and PS ratio optimizing has recently been studied for multi-input single-out (MISO) BC networks $[15,16]$. Recently, the joint transceiver design and power splitting (JTDPS) schemes based on the MSE criterion for MIMO BC channel networks has been investigated in the work of $[17,18]$.

All of the above work is done in single-cell scenarios, where the interaction between the neighbor cells is neglected. In practical wireless communications systems, especially in densely-deployed areas, there exists significant inter-cell interference. Therefore, it is more general and natural to consider SWIPT schemes for interference channel (IC) networks, where multiple transceiver pairs interfere with each other. Some pioneering work on SWIPT for IC networks can be found in [19-21]. Timotheou et al. proposed a SWIPT scheme for MISO IC networks where the receivers are characterized by both quality-of-service (QoS) and EH constraints [19]. The SDR technique was adopted to find the solution of the formulated design problem. Since the computational complexity of the SDP is high when the system becomes large, Shi et al. developed the second-order cone programming (SOCP) techniques based on the system model constructed by [20] . The work in [21] generalized the work of $[19,20]$ by taking the channel imperfection into consideration and proposed robust counterparts of the JTDPS schemes for MISO IC networks. However, these schemes cannot be applied to MIMO IC networks straightforwardly, where the receivers are equipped with multiple antennas. Currently, it is becoming a new research area to investigate the SWIPT schemes for MIMO IC networks [22,23].

Traditionally, interference has be widely recognized as a bottleneck that restricts the capacity scaling of such MIMO IC networks. Consequently, the traditional transceiver designs have put their main effort 
into how to eliminate the interference at the receiver side completely. Interference alignment (IA) is one of the most promising schemes suppressing the interference [24,25]. Through IA, each user can transmit as there is no interference, and the optimal degrees of freedom (DoF) of IC networks can be achieved. The earliest application of IA can be dated back to two decades ago in solving the indexing coding problem [26]. After nearly a decade of silence, IA emerged again in $[27,28]$ and was systematically clarified in $[24,25,29]$. From then on, IA was developed as a general principle for interference management and capacity exploration of multiuser communication systems. What makes IA even more attractive is that it can be realized in the spatial domain via linear transmit precoding and receiver filtering. This means that interference can be eliminated completely at a high signal-to-noise ratio (SNR) regime, and thus, the capacity of the interference channel networks will not be limited by interference anymore.

At present, the applications of IA can be found widely in multiuser MIMO systems, such as MIMO IC networks [30-35], the MIMO interference broadcasting channel (IBC) [36,37], MIMO cognitive networks [38-40], etc. Among these works, the joint transceiver design is the key way to achieve the spacial IA. Since the IA transceiver design problems are over-constrained, the closed-form solutions can be obtained in very limited scenarios $[24,36]$. The most famous closed-form IA solution was proposed in [24] for three-user symmetric MIMO IC networks. The work in [36] derived closed-form solutions for MIMO IBC networks with some special structures. For the networks with general structures on user and antenna numbers, there are generally no closed-form solutions. Alternatively, various iterative methods were proposed in the literature. The work in [30] proposed the famous interference leakage minimization (MIL) and alternative SINR maximization (max-SINR) algorithms. In [31], a subspace-based alternative IA algorithm was derived. The IA schemes based on the mean-square error (MSE) criterion were investigated in [32,33] for MIMO IC networks and in [37] for MIMO IBC networks. In $[34,35]$, the IA transceiver designs were recast into the rank-constrained minimization framework, and the re-weighted nuclear norm minimization algorithms for IA were developed. Different from the aforementioned transceiver design methods where the users collaborate to achieve IA, [38-40] investigated the noncooperative IA scheme in the cognitive networks by opportunistically scheduling the secondary user to align the interference subchannels of the primary user.

However, all of these works focus on either EH or IA separately. In fact, there is a natural correlation between EH and IA with respect to the interference [22,23]. On the one hand, interference is harmful to information transmission. On the other hand, interference is beneficial to EH. Therefore, combing IA with energy harvesting may exploit the potential of both techniques. Recently, some pioneering work on IA-based SWIPT has been proposed in [41,42]. In [41], JTDPS schemes based on IA for K-user MIMO IC networks were investigated. The SDP-based solving methods were developed for single data stream IA systems to obtain the optimal transceivers and PS ratios. The work in [42] analyzed the performance of SWIPT in IA networks and derived lower and upper bounds of harvested power. Power splitting and power allocation optimization algorithms were also proposed in [42] for the case that there is a power splitter.

This work studies further practical simultaneous wireless information and power transfer (SWIPT) schemes for MIMO IC networks, where multiple antennas are equipped not only at transmitters, but also at receivers. The focus of this work is on systems with PS receivers where the received signal is separated into two parts for simultaneously transmitting information and energy harvesting. Note that a closely related work has been proposed in [41] for MIMO IC networks with single data stream transmission, and our work is not a straightforward generation from it. Since for either MIL IA algorithm or the closed-form IA algorithm, only the interference links are considered in the design of the IA transceivers, there exists intra-user interference between the concurrent data streams. This makes the SWIPT schemes proposed in [41] not able to be directly adopted for systems with multiple data stream transmission.

The objective of this work is to jointly deign transmit precoders, receive filters and PS ratios to minimize the total transmit power under both the per-stream SINR threshold and per-receiver energy 
harvesting $(\mathrm{EH})$ threshold constraints. The formulated joint transceiver design and power splitting problem is jointly non-convex with respect to the precoders, information decoding filters and PS ratios and thus difficult to solve optimally. To solve it, the feasibility conditions are firstly analyzed and derived, which guarantee the existence of a solution. Analysis shows that the problem is feasible if the interference can be perfectly suppressed. Considering the difficulty in obtaining the optimal solution for the non-convex problem, an iterative scheme is proposed, which alternatively optimizes the transmitter together with the PS factors and the receivers. The computational complexity of this scheme is high, since the SDP technique is utilized in this scheme to optimize the transmitters and PS factors. To overcome this drawback, a low-complexity design is then proposed by separately designing transceivers to suppress interference using IA and optimizing the transmit power allocation together with receive power splitting. By fixing the precoders and receive filters, the transmit power allocation and receive power splitting optimization problem is then recast as a convex optimization problem and solved optimally. To further reduce the computational complexity, another scheme that has a closed-form solution is proposed for the transmit power allocation and receive PS ratios' optimization. Simulations are done to verify the performance of the proposed schemes, and the results show the effectiveness of all of the proposed solutions. In summary, the main contributions of this work are listed below:

(1) We formulate a design scheme for MIMO SWIPT IC networks where the data transmission operates in spatial complexing mode for each user. The formulated design scheme is more general than the similar schemes studied in the literature, where the MISO networks were considered or only a single data stream is allowed for each user.

(2) We perform a series of theoretical analyses on the feasibility of the design problem. Our analysis reveals that the feasibility of the formulated JTDPS problem is independent of the EH and PS constraints. Based on the analysis, we derive a sufficient condition for the feasibility of the problem. It is thus revealed that an IA feasible network must be feasible for the formulated problem. This makes it possible to check the feasibility of the formulated problem. To the best of our knowledge, there is no similar work to reveal this relationship in the literature.

(3) We derive an iterative scheme based on SDP relaxation techniques to solve the formulated non-convex JTDPS problem. The convergence of the algorithm is proven. An IA-based initializing method is further proposed, which guarantees the convergence of the algorithm.

(4) Based on IA, low-complexity schemes are developed for solving the formulated SWIPT problem more efficiently. Specifically, two transmit power allocation and receive power splitting schemes are developed. Both theoretical analysis and simulations verify the effectiveness of the proposed low-complexity schemes.

Notations: $\mathcal{C}$ represents the complex and positive real field. Bold uppercase and lowercase letters represent matrix and column vectors, respectively. Non-bold italic letters represent scalar values. $\mathbf{I}_{N}$ is an $N \times N$ identity matrix. $\mathbf{A}^{\mathrm{H}}, \mathbf{A}^{\mathrm{T}}$ and $\mathbf{A}^{-1}$ represent the Hermitian transpose, transpose and inverse of $\mathbf{A}$, respectively. $\operatorname{Tr}(\mathbf{A})$ and $\operatorname{rank}(\mathbf{A})$ are the trace and rank of matrix $\mathbf{A}$, respectively. $|\mathbf{A}|$ denotes the determinant of matrix A. $\mathbf{A}=\operatorname{diag}\left(a_{1}, \ldots, a_{i}, a_{i+1}, \ldots, a_{N}\right)$ is a $N \times N$ diagonal matrix with the $i$-th diagonal elements being $a_{i} \cdot \mathbb{E}[\cdot]$ denotes the statistical expectation. $\|\cdot\|_{2}$ and $\|\cdot\|_{\mathrm{F}}$ denote the two- and Frobenius-norm, respectively.

The remainder of this paper is organized as follows. Section 2 introduces the system model and formulates the design problem. The feasibility analysis is provided in Section 3. Section 4 proposes the iterative solving algorithm for the nonconvex problem. The low-complexity designs based on interference alignment are developed in Section 5. Both the optimal and closed-form transmit power allocation and receive power splitting schemes are also provided in this section. Section 6 presents the simulation results. Section 7 concludes our work. 


\section{System Model}

\subsection{MIMO Interference Channel SWIPT System}

We consider a K-user MIMO IC network as shown in Figure 1, where each user consists of a pair of transmitters with $n_{\mathrm{t}}$ transmit antennas and $n_{\mathrm{r}}$ receive antennas. It is assumed that the transceiver pairs share the same frequency band, each operating in SWIPT mode. The channel propagation matrix from transmitter $j$ to receiver $k$ is denoted by $\mathbf{H}_{k j} \in \mathcal{C}^{n_{\mathrm{r}} \times n_{\mathrm{t}}}, \forall k, j \in\{1, \ldots, K\}$. The block fading model is assumed for the channels, which are constant over one transmission frame, but change independently between successive frames, and suppose the elements of $\mathbf{H}_{k j}$ are independent identically distributed (i.i.d.) zero-mean circularly-symmetrical complex Gaussian (ZMCSCG) random variables. $\mathbf{H}_{k k}$ describes the channel coefficients of the desired direct link of the $k$-th user pair, and $\mathbf{H}_{k j}, \forall j \neq k$ constitute channel coefficients of the interference link from the $j$-th transmitter to the $k$-th receiver.

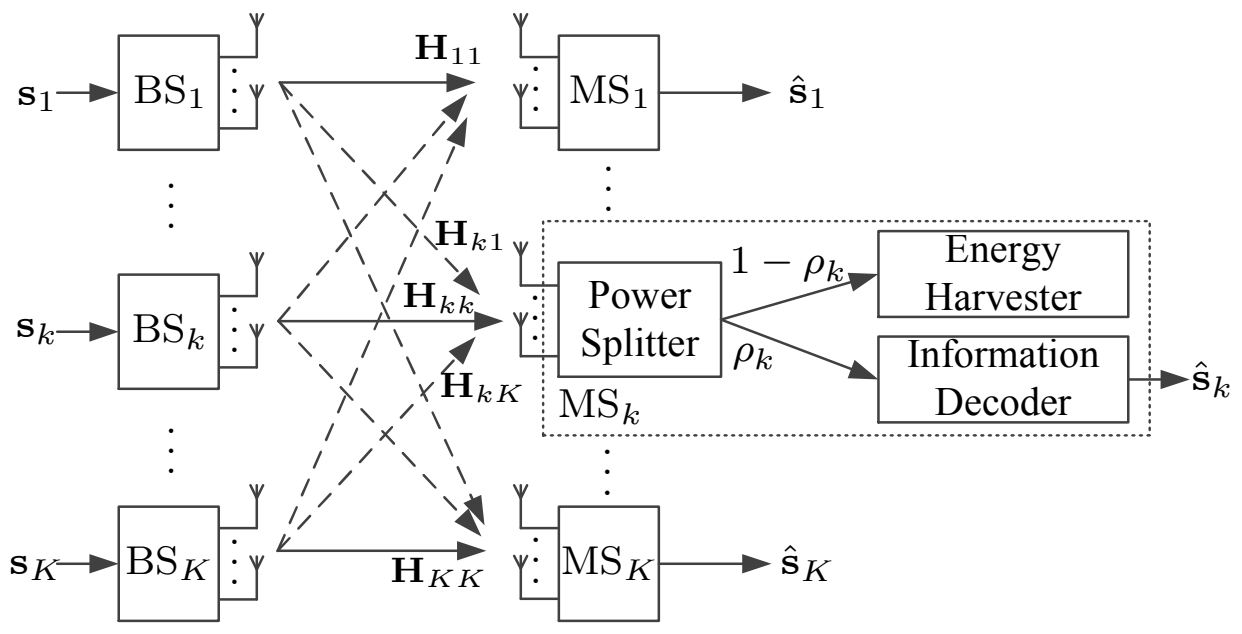

Figure 1. MIMO interference channel SWIPT system.

For simplicity, the considered symmetrical MIMO IC network is henceforth denoted by $\left(n_{\mathrm{t}}, n_{\mathrm{r}}, d\right)^{K}$. The data vector of transmitter $j$ is denoted by $\mathbf{s}_{j} \in \mathcal{C}^{d \times 1}$, and it is assumed $\mathbb{E}\left[\mathbf{s}_{j} \mathbf{s}_{j}^{\mathrm{H}}\right]=\mathbf{I}_{d}$. At the transmit side, the data vector $\mathbf{s}_{j}$ is precoded by transmit precoding matrix $\mathbf{V}_{k} \in \mathcal{C}^{n_{\mathrm{t}} \times d}$ before being sent out. The transmit power of the $k$-th transmitter is assumed to be $\mathbb{E}\left[\operatorname{Tr}\left(\mathbf{V}_{j} \mathbf{V}_{j}^{\mathrm{H}}\right)\right]=P_{j}$. After transmission, the received baseband signals at the $k$-th receiver can be written as:

$$
\mathbf{r}_{k}=\mathbf{H}_{k k} \mathbf{V}_{k} \mathbf{s}_{k}+\sum_{j=1, j \neq k}^{K} \mathbf{H}_{k j} \mathbf{v}_{j} \mathbf{s}_{j}+\mathbf{n}_{k}
$$

where $\mathbf{n}_{k} \in \mathcal{C}^{n_{\mathbf{r} \times 1}}$ denotes the noise vector at the $k$-th receiver. The elements of $\mathbf{n}_{k}$ are assumed to be i.i.d. complex Gaussian random variables with variance $\sigma_{\mathbf{n}_{k}}^{2}$.

The received signal at each antenna is then divided into two parts via a power splitter; one part is used for information decoding, and the other part is transformed to stored energy. In this work, the uniform PS model is adopted [41], which assumes that the adjustable PS factors of a receiver for different antennas are the same such that a scalar PS parameter needs to be optimized. $\rho_{k}$ is defined to be the PS factor for user $k$. The power splitter divides the $\rho_{k}\left(0 \leq \rho_{k} \leq 1\right)$ portion of the received signal into the ID receiver and the remaining $1-\rho_{k}$ portion of the received signal into the EH receiver. Then, the signal split into the ID receiver of the $k$-th user is expressed as:

$$
\mathbf{r}_{k}^{\mathrm{ID}}=\sqrt{\rho_{k}}\left(\mathbf{H}_{k k} \mathbf{V}_{k} \mathbf{s}_{k}+\sum_{j=1, j \neq k}^{K} \mathbf{H}_{k j} \mathbf{V}_{j} \mathbf{s}_{j}+\mathbf{n}_{k}\right)+\mathbf{w}_{k},
$$


where $\mathbf{w}_{k} \sim \mathcal{C N}\left(\mathbf{0}, \sigma_{\mathbf{w}_{k}}^{2} \mathbf{I}_{n_{r}}\right)$ is the additive complex Gaussian noise introduced by the power splitter.

Let $\mathbf{U}_{k}$ denote the receive filter for information decoding at the $k$-th receiver; the recovered signal is expressed as:

$$
\begin{aligned}
\hat{\mathbf{s}}_{k} & =\sqrt{\rho_{k}} \mathbf{U}_{k}^{H} \mathbf{r}_{k}^{I D} \\
& =\underbrace{\sqrt{\rho_{k}} \mathbf{U}_{k}^{H} \mathbf{H}_{k k} \mathbf{V}_{k} \mathbf{s}_{k}}_{\text {Desired Signal }}+\underbrace{\sum_{j=1, j \neq k}^{K} \sqrt{\rho_{k}} \mathbf{U}_{k}^{H} \mathbf{H}_{k j} \mathbf{V}_{j} \mathbf{s}_{j}}_{\text {Interference }}+\underbrace{\sqrt{\rho_{k}} \mathbf{U}_{k}^{H} \mathbf{n}_{k}+\mathbf{U}_{k}^{H} \mathbf{w}_{k}}_{\text {Noise }} .
\end{aligned}
$$

The SINR of the $l$-th data stream of the $k$-th user is defined as:

$$
\operatorname{SINR}_{k l}=\frac{\rho_{k}\left|\mathbf{u}_{k l}^{H} \mathbf{H}_{k k} \mathbf{v}_{k l}\right|^{2}}{\sum_{(j, m) \neq(k, l)} \rho_{k}\left|\mathbf{u}_{k l}^{H} \mathbf{H}_{k j} \mathbf{v}_{j m}\right|^{2}+\left(\rho_{k} \sigma_{n}^{2}+\sigma_{w}^{2}\right) \mathbf{u}_{k l}^{H} \mathbf{u}_{k l}},
$$

where $\mathbf{u}_{k l}$ and $\mathbf{v}_{k l}$ denote the $l$-th column vector in $\mathbf{U}_{k}$ and $\mathbf{V}_{k}$, respectively.

On the other hand, the harvested energy at the $k$-th receiver is:

$$
P_{k}^{\mathrm{EH}}=\xi_{k}\left(1-\rho_{k}\right)\left[\sum_{j=1}^{K} \operatorname{Tr}\left(\mathbf{H}_{k j} \mathbf{V}_{j} \mathbf{V}_{j}^{H} \mathbf{H}_{k j}^{H}\right)+n_{r} \sigma_{\mathbf{n}_{k}}^{2}\right],
$$

where $\xi_{k} \in(0,1]$ denotes the energy conversion efficiency.

With the aim to minimize the transmit power under the given QoS constraints, the joint transceiver design and power splitting problem is formulated as:

$$
\begin{gathered}
\min _{\left\{\mathbf{U}_{k}, \mathbf{v}_{k}, \rho_{k}\right\}} \sum_{k=1}^{K} \sum_{l=1}^{d}\left\|\mathbf{v}_{k l}\right\|_{2}^{2} \\
\text { s.t.: } \quad \operatorname{SINR}_{k l} \geq \gamma_{k l}, \\
\quad P_{k}^{\mathrm{EH}} \geq \psi_{k}, \\
0 \leq \rho_{k} \leq 1, \forall(k, l) .
\end{gathered}
$$

The problem (6) is non-convex due to not only the coupled $\mathbf{u}_{k l}, \mathbf{v}_{k l}$ and $\rho_{k}$ in the QoS constraints (7) and (8), but also all of the quadratic terms including $\mathbf{u}_{k l}$ and $\mathbf{v}_{k l}$; thus, it is very difficult to obtain the optimal solution. Although joint beamforming design and power splitting problems have been investigated recently in $[15,20]$ for MISO IC networks, where the receivers are equipped with only one antenna and thus only the transmit precoders are considered, their solutions cannot be extended to MIMO IC networks.

\subsection{Interference Alignment}

In this subsection, we provide some preliminaries on IA and joint transceiver designs for MIMO IC networks. The basic knowledge of this section constitutes the basis for deriving our work in the following sections.

IA is a ground-breaking interference management method for IC networks. The idea of IA is to coordinate the transmitters so that the interference received at each receiver can be aligned into a subspace with a small dimension and thus leaves the interference-free subspace for signal [24,43]. IA has the ability to achieve the maximum degrees of freedom in a variety of settings, such as the $K$-user IC networks and the symmetric K-user MIMO IC networks [24]. This means that an interference aligned system can transmit as if there were no interference. Besides, one more attractive property of IA is that it can be achieved through linear precoding in MIMO systems. With such linear processing, 
it is possible to realize with lower complexity in practice. In the following, we give a brief introduction to the basic conditions for a symmetric K-user MIMO IC network.

As shown by Figure 1, when the EH receivers are removed, the system degenerates into traditional symmetric MIMO IC networks. With the assumption of generic channel matrices (a channel matrix is generic, which means that its elements are drawn independently from a continuous probability distribution, such that the probability that the matrix is rank-deficient is zero almost surely), the DoF of this system is $\min \left(n_{t}, n_{r}\right) K / 2$ [24]. The system can achieve the linear IA only if the following feasibility conditions are satisfied [30].

$$
\begin{gathered}
\mathbf{U}_{k}^{\mathrm{H}} \mathbf{H}_{k j} \mathbf{V}_{j}=\mathbf{0}, \forall j, k \in\{1, \ldots, K\}, j \neq k \\
\operatorname{rank}\left(\mathbf{U}_{k}^{\mathrm{H}} \mathbf{H}_{k k} \mathbf{V}_{k}\right)=d,
\end{gathered}
$$

The condition (10) requires that the interference should be suppressed completely, while the condition (11) guarantees that the signal dimension left for each user is $d$. For the considered system, in order to achieve IA, the maximum number of streams for each user should be constrained by $d \leq\left(n_{t}+n_{r}\right) /(K+1)[44]$.

If the IA condition is perfectly satisfied, the interference term in (1) can be completely suppressed. In this situation, the recovered signal at receiver $k$ reduces to:

$$
\begin{aligned}
\hat{\mathbf{s}}_{k} & =\mathbf{U}_{k}^{\mathrm{H}} \mathbf{r}_{k} \\
& =\mathbf{U}_{k}^{\mathrm{H}} \mathbf{H}_{k k} \mathbf{V}_{k} \mathbf{x}_{k}+\mathbf{U}_{k}^{\mathrm{H}} \mathbf{n}_{k} \\
& =\overline{\mathbf{H}}_{k} \mathbf{s}_{k}+\overline{\mathbf{n}}_{k},
\end{aligned}
$$

where $\overline{\mathbf{H}}_{k}=\mathbf{U}_{k}^{\mathrm{H}} \mathbf{H}_{k k} \mathbf{V}_{k}$ and $\overline{\mathbf{n}}_{k}=\mathbf{U}_{k}^{\mathrm{H}} \mathbf{n}_{k}$ denote the effective channel matrix and the effective noise vector at receiver $k$, respectively. Given perfect global CSI, $\overline{\mathbf{n}}_{k}$ is a Gaussian vector with covariance $\mathbb{E}\left[\overline{\mathbf{n}}_{k} \overline{\mathbf{n}}_{k}^{\mathrm{H}}\right]=\sigma_{\mathbf{n}_{k}} \mathbf{I}_{d}$, and it is uncorrelated with $\mathbf{s}_{k}$ [45]. Then, (12) means that the system can be equivalent to a point-to-point MIMO system after IA, and the ergodic achievable rate for the $k$-th user is written as:

$$
\mathcal{R}_{k}=\mathbb{E}\left[\log _{2}\left|\mathbf{I}_{d}+\frac{\overline{\mathbf{H}}_{k} \overline{\mathbf{H}}_{k}^{\mathrm{H}}}{\sigma_{\mathbf{n}_{k}^{2}}}\right|\right] .
$$

However, the IA feasibility condition (10) is over-constrained on the transceivers [24,46], and it is not trivial to obtain its solution. Till now, there have been very rare situations in which the closed-form solution can be derived. For example, the closed-form IA solution can be obtained for a symmetric system with three users. However, if the number of users becomes larger than three, it is very difficult to obtain a closed-form solution. Therefore, the more general and efficient methods to obtain the IA solutions are the iterative algorithms, such as the MIL algorithm [30], the max-SINR algorithm [30], the MMSE algorithm [33], etc.

In the following sections, the feasibility of the formulated problem (6) will be discussed, and suboptimal schemes solving the problem will be developed.

\section{On the Feasibility of the Optimization Problem}

When the receivers are equipped with a single antenna, Problem (6) degenerates to the traditional joint beamforming and PS problem for MISO IC networks, the feasibility of which has been well investigated in $[15,20,21]$. For an MISO IC network, a basic observation is that the feasibility of problem (6) is independent of the EH constraints and PS ratios. This observation can be generalized to MIMO IC systems, which is given by the following lemma. 
Lemma 1. Problem (6) is feasible if and only if the following problem is feasible.

$$
\begin{aligned}
\text { Find: } & \left\{\mathbf{U}_{k}, \mathbf{V}_{k}, \rho_{k}\right\} \\
\text { Such that: } & \operatorname{SINR}_{k l} \geq \gamma_{k l}, \\
& 0 \leq \rho_{k} \leq 1, \forall(k, l) .
\end{aligned}
$$

Proof. Suppose that Problem (14) is not feasible; it can be verified that Problem (6) is not feasible. This is because there exist additional constraints on EH in Problem (6) compared with Problem (14).

On the other hand, assume Problem (14) is feasible, and let $\left\{\mathbf{U}_{k}, \mathbf{V}_{k}, \rho_{k}, \forall k\right\}$ be a feasible solution; for any $\alpha>1$, the solution $\left\{\mathbf{U}_{k}, \alpha \mathbf{V}_{k}, \rho_{k}, \forall k\right\}$ is also feasible. That is to say that the achieved SINR does not decrease if all data streams scale up their power at the same rate. According to (5), the harvested energy can always increase together with the booming up transmit power. Therefore, if Problem (14) is feasible, then there is always an $\alpha$ that is large enough to satisfy the EH constraints in (6).

Lemma 1 shows that the feasibility of Problem (6) is independent of the EH constraints. The following lemma shows that the feasibility of Problem (6) is also independent of the PS factors.

Lemma 2. Problem (14) is feasible if and only if the following problem is feasible.

Find: $\left\{\mathbf{U}_{k}, \mathbf{V}_{k}\right\}$

Such that: $\operatorname{SINR}_{k l}^{\prime} \geq \gamma_{k l}, \forall(k, l)$,

where:

$$
\operatorname{SINR}_{k l}^{\prime}=\frac{\left|\mathbf{u}_{k l}^{H} \mathbf{H}_{k k} \mathbf{v}_{k l}\right|^{2}}{\sum_{(j, m) \neq(k, l)}\left|\mathbf{u}_{k l}^{H} \mathbf{H}_{k j} \mathbf{v}_{j m}\right|^{2}+\left(\sigma_{n}^{2}+\sigma_{w}^{2}\right) \mathbf{u}_{k l}^{H} \mathbf{u}_{k l}} .
$$

Proof. Suppose that Problem (15) is feasible, and let $\left\{\mathbf{U}_{k}, \mathbf{V}_{k}, \forall k\right\}$ be a feasible solution; given an arbitrary power splitting factor $0<\rho<1$, if the transmit precoders are scaled as $\left\{\mathbf{V}_{k} / \sqrt{\rho}, \forall k\right\}$, the SINR for the $l$-th data stream of the $k$-th user is written as:

$$
\begin{aligned}
\operatorname{SINR}_{k l} & =\frac{\rho\left|\mathbf{u}_{k l}^{H} \mathbf{H}_{k k} \mathbf{v}_{k l} / \sqrt{\rho}\right|^{2}}{\sum_{(j, m) \neq(k, l)} \rho\left|\mathbf{u}_{k l}^{H} \mathbf{H}_{k j} \frac{\mathbf{v}^{m}}{\sqrt{\rho}}\right|^{2}+\left(\rho \sigma_{n}^{2}+\sigma_{w}^{2}\right) \mathbf{u}_{k l}^{H} \mathbf{u}_{k l}} \\
& =\frac{\left|\mathbf{u}_{k l}^{H} \mathbf{H}_{k k} \mathbf{v}_{k l}\right|^{2}}{\sum_{(j, m) \neq(k, l)}\left|\mathbf{u}_{k l}^{H} \mathbf{H}_{k j} \mathbf{v}_{j m}\right|^{2}+\left(\rho \sigma_{n}^{2}+\sigma_{w}^{2}\right) \mathbf{u}_{k l}^{H} \mathbf{u}_{k l}} \\
& >\frac{\left|\mathbf{u}_{k l}^{H} \mathbf{H}_{k k} \mathbf{v}_{k l}\right|^{2}}{\sum_{(j, m) \neq(k, l)}\left|\mathbf{u}_{k l}^{H} \mathbf{H}_{k j} \mathbf{v}_{j m}\right|^{2}+\left(\sigma_{n}^{2}+\sigma_{w}^{2}\right) \mathbf{u}_{k l}^{H} \mathbf{u}_{k l}} \\
& =\operatorname{SINR}_{k l}^{\prime} \geq \gamma_{k l} .
\end{aligned}
$$

Therefore, the solution $\left\{\mathbf{U}_{k}, \mathbf{V}_{k} / \sqrt{\rho}, \rho, \forall k\right\}$ is feasible to Problem (14).

If Problem (15) is not feasible, it can be proven that Problem (14) is not feasible by contradiction as follows. Suppose that Problem (14) is feasible, and let $\left\{\mathbf{U}_{k}, \mathbf{V}_{k}, \rho_{k}, \forall k\right\}$ be a feasible solution. Since $\rho_{k} \leq 1$, it holds that: 


$$
\begin{aligned}
\gamma_{k l} & \leq \frac{\rho_{k}\left|\mathbf{u}_{k l}^{H} \mathbf{H}_{k k} \mathbf{v}_{k l}\right|^{2}}{\sum_{(j, m) \neq(k, l)} \rho_{k}\left|\mathbf{u}_{k l}^{H} \mathbf{H}_{k j} \mathbf{v}_{j m}\right|^{2}+\left(\rho_{k} \sigma_{n}^{2}+\sigma_{w}^{2}\right) \mathbf{u}_{k l}^{H} \mathbf{u}_{k l}} \\
& =\frac{\left|\mathbf{u}_{k l}^{H} \mathbf{H}_{k k} \mathbf{v}_{k l}\right|^{2}}{\sum_{(j, m) \neq(k, l)}\left|\mathbf{u}_{k l}^{H} \mathbf{H}_{k j} \mathbf{v}_{j m}\right|^{2}+\left(\sigma_{n}^{2}+\frac{\sigma_{w}^{2}}{\rho_{k}}\right) \mathbf{u}_{k l}^{H} \mathbf{u}_{k l}} \\
& \leq \frac{\left|\mathbf{u}_{k l}^{H} \mathbf{H}_{k k} \mathbf{v}_{k l}\right|^{2}}{\sum_{(j, m) \neq(k, l)}\left|\mathbf{u}_{k l}^{H} \mathbf{H}_{k j} \mathbf{v}_{j m}\right|^{2}+\left(\sigma_{n}^{2}+\sigma_{w}^{2}\right) \mathbf{u}_{k l}^{H} \mathbf{u}_{k l}} \\
& =\operatorname{SINR}_{k l}^{\prime} .
\end{aligned}
$$

Thus, $\left\{\mathbf{U}_{k}, \mathbf{V}_{k}, \rho_{k}, \forall k\right\}$ is also a feasible solution for Problem (15), which contradicts with the assumption that Problem (15) is not feasible.

From Lemma 2, it becomes easy to check the feasibility of the original problem. Moreover, for an MISO IC network, Problem (15) can be solved efficiently by using the uplink-downlink duality $[20,47,48]$. For the MIMO IC network, the feasibility of Problem (15) is an open problem, and it is very difficult to find the solution of Problem (15), since it is a non-convex and NP-hard problem [41,49]. In the following proposition, a sufficient condition for the feasibility of Problem (15) is presented based on IA.

Proposition 1. Problem (15) is feasible for any given SINR constraints if the system is interference unlimited, i.e., the interference can be completely eliminated by the linear transceivers.

Proof. If interference is completed eliminated, given the transceivers $\mathbf{U}_{k}, \mathbf{V}_{k}, \forall k$, i.e.,

$$
\begin{gathered}
\mathbf{u}_{k l}^{\mathrm{H}} \mathbf{H}_{k j} \mathbf{v}_{j m}=\mathbf{0}, \forall(j, m) \neq(k, l), \\
\mathbf{u}_{k l}^{\mathrm{H}} \mathbf{H}_{k k} \mathbf{v}_{k l} \neq 0, \forall(k, l),
\end{gathered}
$$

the SINR (16) becomes:

$$
\operatorname{SINR}_{k l}^{\prime}=\frac{\left|\mathbf{u}_{k l}^{\mathrm{H}} \mathbf{H}_{k k} \mathbf{v}_{k l}\right|^{2}}{\left(\sigma_{n}^{2}+\sigma_{w}^{2}\right) \mathbf{u}_{k l}^{\mathrm{H}} \mathbf{u}_{k l}}=\frac{p_{k l}\left|\mathbf{u}_{k l}^{\mathrm{H}} \mathbf{H}_{k k} \overline{\mathbf{v}}_{k l}\right|^{2}}{\left(\sigma_{n}^{2}+\sigma_{w}^{2}\right)\left\|\mathbf{u}_{k l}\right\|_{2}^{2}},
$$

where $\overline{\mathbf{v}}_{k l}=\frac{\mathbf{v}_{k l}}{\left\|\mathbf{v}_{k l}\right\|_{2}}$ is the normalized precoding vector, $p_{k l}$ is the transmit power along the beamforming direction $\overline{\mathbf{v}}_{k l}$ and $\mathbf{v}_{k l}=p_{k l} \overline{\mathbf{v}}_{k l}$. According to (21), the SINR constraints in Problem (15) can always be satisfied by increasing the transmit power $p_{k l}$, if the interference is completely suppressed.

To achieve Condition (19), the numbers of antenna and the data stream for each user in the MIMO IC network should be strictly constrained. One special case that can satisfy Condition (19) is the IA network. If the antennas and data streams are configured such that the linear interference alignment is achievable, then there exist IA precoders $\mathbf{V}_{k}^{\mathrm{IA}}, \forall k$ and filters $\left\{\mathbf{U}_{k}^{\mathrm{IA}}, \forall k\right\}[24,50-52]$ to satisfy the conditions in (22), i.e.,

$$
\begin{gathered}
\left(\mathbf{U}_{k}^{\mathrm{IA}}\right){ }^{\mathrm{H}} \mathbf{H}_{k j} \mathbf{V}_{j}^{\mathrm{IA}}=\mathbf{0}, \forall j, k \in\{1, \ldots, K\}, j \neq k, \\
\operatorname{rank}\left[\left(\mathbf{U}_{k}^{\mathrm{IA}}\right)^{\mathrm{H}} \mathbf{H}_{k k} \mathbf{V}_{k}^{\mathrm{IA}}\right]=d, \forall k .
\end{gathered}
$$

Condition (22) indicates that the interferences are completely eliminated after IA; while Condition (23) ensures the available signal dimensions for data streams. 
Based on Proposition 1 and the IA feasibility Condition (22), we can infer that Problem (6) must be feasible if the system is IA feasible. In our following work, we assume that the antennas and data streams of the investigated MIMO IC networks are configured to be IA feasible.

\section{Alternative Optimization Solution Based on Semidefinite Programming Relaxation}

Even if it is known that Problem (6) is feasible, it is still very difficult to obtain the optimal solution to the non-convex problem by jointly optimizing all of the variables in Problem (6). Instead of attempting to obtain its global optimal solution, schemes are developed in the following sections to find its near-optimal solution.

With the assumption of feasible IA, according to Proposition 1, Problem (6) must be feasible. To solve it, the alternative optimization method can be applied by alternatively optimizing the transmitters $\mathbf{V}_{k}, \forall k$ together with the PS factors $\rho_{k}, \forall k$ and the receivers $\mathbf{U}_{k}, \forall k$.

\subsection{Transmitter and Power Splitting Optimization}

By fixing the receivers, Problem (6) is reduced to the joint transmit precoders and PS factors optimization problem:

$$
\begin{array}{ll} 
& \min _{\left\{\mathbf{v}_{k l}, \rho_{k}, \forall(k, l)\right\}} \sum_{k=1}^{K} \sum_{l=1}^{d}\left\|\mathbf{v}_{k l}\right\|_{2}^{2} \\
\text { s.t.: } & \frac{\left|\mathbf{u}_{k l}^{H} \mathbf{H}_{k k} \mathbf{v}_{k l}\right|^{2}}{\mathbf{u}_{k l} \mathbf{B}_{k l} \mathbf{u}_{k l}^{H}} \geq \gamma_{k l}, \\
& \sum_{j=1}^{K} \sum_{m=1}^{d}\left\|\mathbf{H}_{k j} \mathbf{v}_{j m}\right\|_{2}^{2} \geq \frac{\psi_{k}}{\xi_{k}\left(1-\rho_{k}\right)}-n_{\mathrm{r}} \sigma_{n}^{2}, \\
& 0 \leq \rho_{k} \leq 1, \forall(k, l),
\end{array}
$$

where $\mathbf{B}_{k l}=\sum_{j=1}^{K} \sum_{m=1}^{d} \mathbf{H}_{k j} \mathbf{v}_{j m} \mathbf{v}_{j m}^{\mathrm{H}} \mathbf{H}_{k j}^{\mathrm{H}}-\mathbf{H}_{k k} \mathbf{v}_{k l} \mathbf{v}_{k l}^{\mathrm{H}} \mathbf{H}_{k k}^{\mathrm{H}}+\left(\sigma_{n}^{2}+\frac{\sigma_{w}^{2}}{\rho_{k}}\right) \mathbf{I}_{n_{\mathrm{r}}}$.

Problem (24) must be feasible according to Lemma 2, Proposition 1 and [53], Proposition 1. The SDP relaxation technique [54] can be used to solve Problem (24). By defining $\mathbf{X}_{k l}=\mathbf{v}_{k l} \mathbf{v}_{k l}^{\mathrm{H}}, \mathbf{X}_{k l} \succeq \mathbf{0}$, Problem (24) can be relaxed as:

$$
\begin{array}{ll} 
& \min _{\left\{\mathbf{x}_{k l}, \rho_{k}, \forall(k, l)\right\}} \sum_{k=1}^{K} \sum_{l=1}^{d} \operatorname{Tr}\left(\mathbf{X}_{k l}\right) \\
\text { s.t.: } & \left(1+\gamma_{k l}\right) \operatorname{Tr}\left(\mathbf{u}_{k l}^{\mathrm{H}} \mathbf{H}_{k k} \mathbf{X}_{k l} \mathbf{H}_{k k}^{\mathrm{H}} \mathbf{u}_{k l}\right) \\
& -\gamma_{k l} \sum_{j=1}^{K} \sum_{m=1}^{d} \operatorname{Tr}\left(\tilde{\mathbf{u}}_{k l}^{\mathrm{H}} \mathbf{H}_{k j} \mathbf{X}_{j m} \mathbf{H}_{k j}^{\mathrm{H}} \mathbf{u}_{k l}\right) \geq \gamma_{k l}\left(\sigma_{n}^{2}+\frac{\sigma_{w}^{2}}{\rho_{k}}\right)\left\|\mathbf{u}_{k l}\right\|_{2}^{2}, \\
& \sum_{j=1}^{K} \sum_{m=1}^{d} \operatorname{Tr}\left[\mathbf{H}_{k j} \mathbf{x}_{j m} \mathbf{H}_{k j}^{\mathrm{H}}\right] \geq \frac{\psi_{k}}{\xi_{k}\left(1-\rho_{k}\right)}-n_{\mathrm{r}} \sigma_{n}^{2}, \\
& 0 \leq \rho_{k} \leq 1, \forall(k, l) .
\end{array}
$$

Problem (25) is a convex SDP problem, which can be solved by a common optimization package designed to solve SDP, such as CVX [55]. It is noted that (34) is convex because both functions $\frac{1}{x}$ and $\frac{1}{1-x}$ are convex with respect to $0<x<1$. It can be proven that the SDP relaxation is tight at the optimal solution such that $\operatorname{rank}\left(\mathbf{X}_{k l}\right)=1$ [41]. Once the rank-one solution $\left\{\mathbf{X}_{k l}, \forall(k, l)\right\}$ has been successfully obtained, the optimal solution $\mathbf{v}_{k l}$ to Problem (24) can then be recovered from solution $\mathbf{X}_{k l}$ via eigenvalue decomposition. 


\subsection{Receiver Optimization}

By fixing the transmitters and PS factors, Problem (6) becomes separable on the variables $\left\{\mathbf{u}_{k l}, \forall(k, l)\right\}$. Since the SINR constraints have been satisfied by the optimized transmitters $\mathbf{v}_{k l}, \forall(k, l)$ and PS factors $\rho_{k}, \forall k$ obtained from the solution of (24), to further optimize the receive per-stream SINR by optimizing the receive vector for each data stream, a maximum SINR problem is then formulated:

$$
\max _{\mathbf{u}_{k l}} \frac{\mathbf{u}_{k l}^{\mathrm{H}} \mathbf{H}_{k k} \mathbf{v}_{k l} \mathbf{v}_{k l}^{\mathrm{H}} \mathbf{H}_{k k}^{\mathrm{H}} \mathbf{u}_{k l}}{\mathbf{u}_{k l}^{\mathrm{H}} \mathbf{B}_{k l} \mathbf{u}_{k l}}
$$

where $\mathbf{B}_{k l}=\sum_{j=1}^{K} \sum_{m=1}^{d} \mathbf{H}_{k j} \mathbf{v}_{j m} \mathbf{v}_{j m}^{\mathrm{H}} \mathbf{H}_{k j}^{\mathrm{H}}-\mathbf{H}_{k k} \mathbf{v}_{k l} \mathbf{v}_{k l}^{\mathrm{H}} \mathbf{H}_{k k}^{\mathrm{H}}+\left(\sigma_{n}^{2}+\frac{\sigma_{w w}^{2}}{\rho_{k}}\right) \mathbf{I}_{n_{\mathrm{r}}}$. Problem (26) is a generalized Rayleigh quotient, the solution of which is given by:

$$
\mathbf{u}_{k l}=\frac{\mathbf{B}_{k l}^{-1} \mathbf{H}_{k k} \mathbf{v}_{k l}}{\left\|\mathbf{B}_{k l}^{-1} \mathbf{H}_{k k} \mathbf{v}_{k l}\right\|_{2}} .
$$

\subsection{Algorithm Description}

By alternatively optimizing the transmitters together with PS factors and the receivers, an SDP-based joint transceiver design and PS scheme are obtained and summarized in Algorithm 1. The convergence of Algorithm 1 is given by the following proposition.

Proposition 2. If Problem (25) is feasible for the initial receivers $\mathbf{U}_{k}, \forall k$, the convergence to a local optimal solution can be guaranteed by Algorithm 1.

Proof. Define $n=0,1,2, \ldots$, as the iteration number, where $n=0$ denotes the initializing step. Let $P(n)$ denote the optimized total transmit power value of Problem (1) at the $n$-th iteration. Denote $\operatorname{SINR}_{k l}\left[\mathbf{V}_{k}(n), \rho_{k}(n) ; \mathbf{U}_{k}(n-1)\right]$ and $\operatorname{SINR}_{k l}\left[\mathbf{V}_{k}(n), \rho_{k}(n) ; \mathbf{U}_{k}(n)\right]$ as the MSE achieved after Steps (3) and (4) of Algorithm 1 for user $k$ at the $n$-th iteration, respectively.

At the first iteration, suppose Problem (25) is feasible given the initial receivers $\mathbf{U}_{k}(0), \forall k$, then there exists a solution $\left\{\mathbf{G}(1), \rho_{k}(1), \forall k\right\}$ after Step (3) of Algorithm 1, such that:

$$
\operatorname{SINR}_{k l}\left[\mathbf{V}_{k}(1), \rho_{k}(1) ; \mathbf{U}_{k}(0)\right] \geq \gamma_{k}
$$

After Step (4) of Algorithm 1, the receivers $\mathbf{U}_{k}(1)$ are optimized to maximize the received SINR. The achieved SINR will not increase in this step, which gives:

$$
\begin{aligned}
\operatorname{SINR}_{k}\left[\mathbf{V}_{k}(1), \rho_{k}(1) ; \mathbf{U}_{k}(1)\right] & \geq \operatorname{SINR}_{k}\left[\mathbf{V}_{k}(1), \rho_{k}(1) ; \mathbf{U}_{k}(0)\right] \\
& \geq \gamma_{k} .
\end{aligned}
$$

Since the update of ID receivers $\mathbf{U}_{k}, \forall k$ does not affect the EH constraints, the solution $\left\{\mathbf{U}_{k}(1), \rho_{k}(1), \mathbf{V}_{k}(1), \forall k\right\}$ is still feasible for Problem (25).

At the second iteration, by fixing the receiver $\mathbf{U}_{k}(1)$, the transmit precoder and PS factors $\left\{\mathbf{V}_{k}(2), \mathfrak{x}_{k}(2), \forall k\right\}$ are optimized after Step (3) of Algorithm 1. Since the solution $\left\{\mathbf{G}(1), \rho_{k}(1), \mathbf{F}_{k}(1), \forall k\right\}$ is feasible for Problem (25), the optimized transmit power will not increase, which leads to:

$$
P(2) \leq P(1)
$$

In the same way, at the $n$-th iteration, we can infer that the solution $\left\{\mathbf{V}_{k}(n), \rho_{k}(n), \mathbf{U}_{k}(n), \forall k\right\}$ is feasible for Problem (25), and the objective transmit power will not increase as the iteration increases, 
i.e., $P(n) \leq P(n-1)$, showing that the proposed Algorithm 1 can converge to a local minimum. The proof is then concluded.

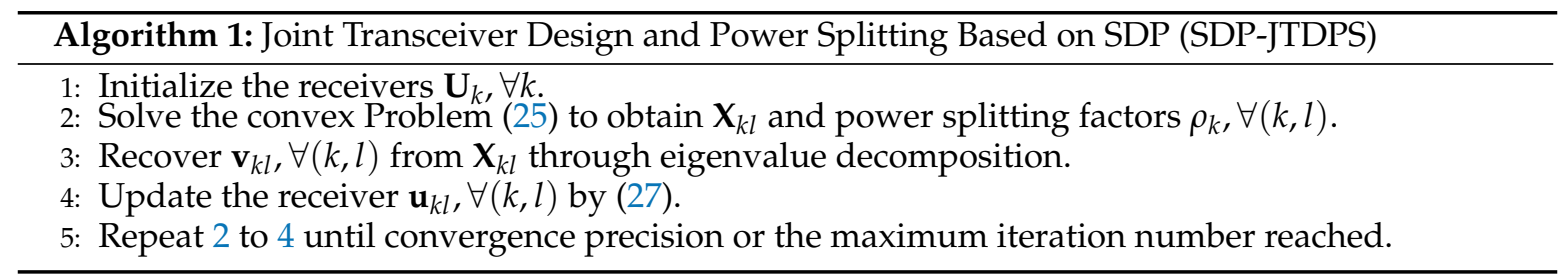

According to Proposition 2, the selection of proper initial receivers $\mathbf{U}_{k}, \forall k$ is of great importance to make Problem (25) and thus Algorithm 1 feasible. A casual initializing, such as generating the initial receivers randomly, can cause Problem (25) to be immediately infeasible, since the inter-user interference can be significant to make the SINR constraints violated. To guarantee finding a feasible solution to Problem (25), we initialize the receivers by the IA receivers $\mathbf{U}_{k}^{\mathrm{IA}}, \forall k$. Our simulations show that initializing the receivers by IA receivers can make Problem (25) feasible.

The complexity of Algorithm 1 is dominated by the SDP solving process for Problem (25). The complexity to solve one instance of Problem (25) is about $\mathcal{O}\left(\left(n_{r} K d\right)^{4.5} \log (1 / \epsilon)\right)$ [54], where $\epsilon>0$ is the given solution accuracy. For Algorithm 1, the computational complexity is estimated as $\mathcal{O}\left(n_{\text {iter }}\left(n_{r} K d\right)^{4.5} \log (1 / \epsilon)\right)$, where $n_{\text {iter }}$ is the iteration number of the algorithm. When the system becomes large in antenna number and user number, the computational complexity of Algorithm 1 is prohibitive. This poses a huge restriction for practical implementation of Algorithm 1. To break these limitations, we propose two low-complexity design schemes in the following section.

\section{Low-Complexity Design Schemes}

We propose two kinds of low complexity schemes to solve Problem (6) by separately designing the transceivers and power splitting factors. The transceivers are first designed by eigen-decomposing the effective channel matrices generated by interference alignment. Then, the transmit power and receive PS factors are optimized with the precoders and receivers fixed.

\subsection{Transceiver Design}

As analyzed in the previous section, to ensure that Problem (6) is feasible, perfect IA should be realized. Without loss of generality and in order to simplify the system design, we assume that the precoders and receive filters are orthogonalized such that $\left(\mathbf{V}_{k}^{\mathrm{IA}}\right)^{\mathrm{H}} \mathbf{V}_{k}^{\mathrm{IA}}=\mathbf{I}_{d}$ and $\left(\mathbf{U}_{k}^{\mathrm{IA}}\right)^{\mathrm{H}} \mathbf{U}_{k}^{\mathrm{IA}}=\mathbf{I}_{d}$. Given the interference alignment transceivers, the effective channel matrix for user $k$ can be decomposed as $\overline{\mathbf{H}}_{k k}=\left(\mathbf{U}_{k}^{\mathrm{IA}}\right)^{\mathrm{H}} \mathbf{H}_{k k} \mathbf{V}_{k}^{\mathrm{IA}}=\overline{\mathbf{U}}_{k} \boldsymbol{\Lambda}_{k} \overline{\mathbf{V}}_{k}^{\mathrm{H}}$ through singular value decomposition (SVD), where $\boldsymbol{\Lambda}_{k}=\operatorname{diag}\left(\sqrt{\lambda_{k 1}}, \sqrt{\lambda_{k 2}}, \ldots, \sqrt{\lambda}_{k d}\right)$ is a diagonal matrix.

The transmit precoder matrix is constructed by cascading the IA precoders and the right eigen-matrix of the effective matrix, i.e., $\mathbf{V}_{k}^{\prime}=\mathbf{V}_{k}^{\mathrm{IA}} \overline{\mathbf{V}}_{k}$. Note that $\mathbf{V}_{k}^{\prime}$ is a unitary matrix. The transmit power is then injected to the precoding vector $\mathbf{V}_{k}^{\prime}$ via power allocation matrices, i.e., $\mathbf{V}_{k}=\mathbf{V}_{k}^{\prime} \mathbf{P}_{k}$, where $\mathbf{P}_{k}=\operatorname{diag}\left(\sqrt{p_{k 1}}, \ldots, \sqrt{p_{k d}}\right)$ is a diagonal matrix with nonnegative diagonalized elements. At the receive side, a zero-forcing filtering is further cascaded to the IA filter. The final receive filter is a unitary matrix and can be denoted as $\mathbf{U}_{k}=\mathbf{U}_{k}^{\mathrm{IA}} \overline{\mathbf{U}}_{k}$. Then, (17) is equivalent to:

$$
\begin{gathered}
\mathbf{U}_{k}^{\mathrm{H}} \mathbf{H}_{k j} \mathbf{V}_{j}=\mathbf{0}, \forall j, k \in\{1, \ldots, K\}, j \neq k, \\
\mathbf{U}_{k}^{\mathrm{H}} \mathbf{H}_{k k} \mathbf{V}_{k}=\boldsymbol{\Lambda}_{k} \mathbf{P}_{k}, \forall k .
\end{gathered}
$$

It can be observed from (31) that interference is completely suppressed at the ID receiver by the proposed transceiver design scheme. According to Lemma 1, Lemma 2 and Proposition 1, we can infer that Problem (6) is feasible if IA is feasible. 


\subsection{Transmit Power Allocation and Receive Power Splitting}

Given the IA precoders and receive filters $\left\{\mathbf{U}_{k}^{\mathrm{IA}}, \mathbf{V}_{k}^{\mathrm{IA}}, \forall k\right\}$, Problem (6) reduces to the following transmit power allocation and receive power splitting problem:

$$
\begin{array}{ll} 
& \min _{\left\{p_{k l}, \rho_{k}, \forall k, l\right\}} \sum_{k=1}^{K} \sum_{l=1}^{d} p_{k l} \\
\text { s.t.: } & \frac{\rho_{k} \lambda_{k l} p_{k l}}{\left(\rho_{k} \sigma_{n}^{2}+\sigma_{w}^{2}\right)\left\|\mathbf{u}_{k l}\right\|_{2}^{2}} \geq \gamma_{k l}, \\
& \xi_{k}\left(1-\rho_{k}\right)\left(\sum_{j=1}^{K} \sum_{m=1}^{d} p_{j m}\left\|\mathbf{H}_{k j} \mathbf{v}_{j m}^{\prime}\right\|_{2}^{2}+n_{\mathrm{r}} \sigma_{n}^{2}\right) \geq \psi_{k}, \\
& 0 \leq \rho_{k} \leq 1, \forall(k, l) .
\end{array}
$$

Problem (33) is still non-convex in its current form. In the following, we provide two schemes to solve Problem (33).

\subsection{Low-Complexity Designs}

Problem (33) can be either reformulated as a convex problem or solved in closed-form. For the convex optimization scheme, the optimal power allocation for data streams and power splitting for EH can be obtained. For the closed-form solution, the power allocation and splitting are suboptimal, but can be calculated with very low complexity.

5.3.1. Optimal Power Allocation and Power Splitting Scheme

Problem (33) can be further transformed to be convex as follows:

$$
\begin{array}{ll} 
& \min _{\left\{p_{k l}, \rho_{k}, \forall k, l\right\}} \sum_{k=1}^{K} \sum_{l=1}^{d} p_{k l}\left\|\mathbf{v}_{k l}^{\mathrm{IA}}\right\|_{2}^{2} \\
\text { s.t.: } & p_{k l}-\frac{\sigma_{n}^{2} \gamma_{k l}\left\|\mathbf{u}_{k l}\right\|_{2}^{2}}{\lambda_{k l}} \geq \frac{\sigma_{w}^{2}\left\|\mathbf{u}_{k l}\right\|_{2}^{2} \gamma_{k l}}{\lambda_{k l} \rho_{k}}, \\
& \sum_{j=1}^{K} \sum_{m=1}^{d} p_{j m}\left\|\mathbf{H}_{k j} \mathbf{v}_{j m}^{\prime}\right\|_{2}^{2} \geq \frac{\psi_{k}}{\xi_{k}\left(1-\rho_{k}\right)}-n_{\mathrm{r}} \sigma_{n}^{2}, \\
& 0 \leq \rho_{k} \leq 1, \forall(k, l) .
\end{array}
$$

Problem (34) is now convex considering that the objective and all constraints are convex, and it can be solved in polynomial time via interior point methods. In this paper, we obtain the transmit power and PS factors efficiently by solving Problem (34) using convex optimization toolboxes [55]. Let $p_{k l}^{*}$ and $\rho_{k}^{*}, \forall k$ denote the optimal solution of (34), the transmit precoders are then determined by $\mathbf{v}_{k l}=\sqrt{p_{k l}^{*}} \mathbf{v}_{k l}^{\mathrm{IA}}$.

The proposed IA-based SWIPT scheme with optimal power allocation and power splitting is summarized in Algorithm 2.

The computational complexity of Algorithm 2 is mainly expended in the computing of Problem (34) in Step 4. If the interior methods are employed to solve Problem (34), the computational complexity of Algorithm 2 is in the order of $\mathcal{O}\left((K d)^{3}\right)$ [56], which is significantly lower than that of Algorithm 1. 


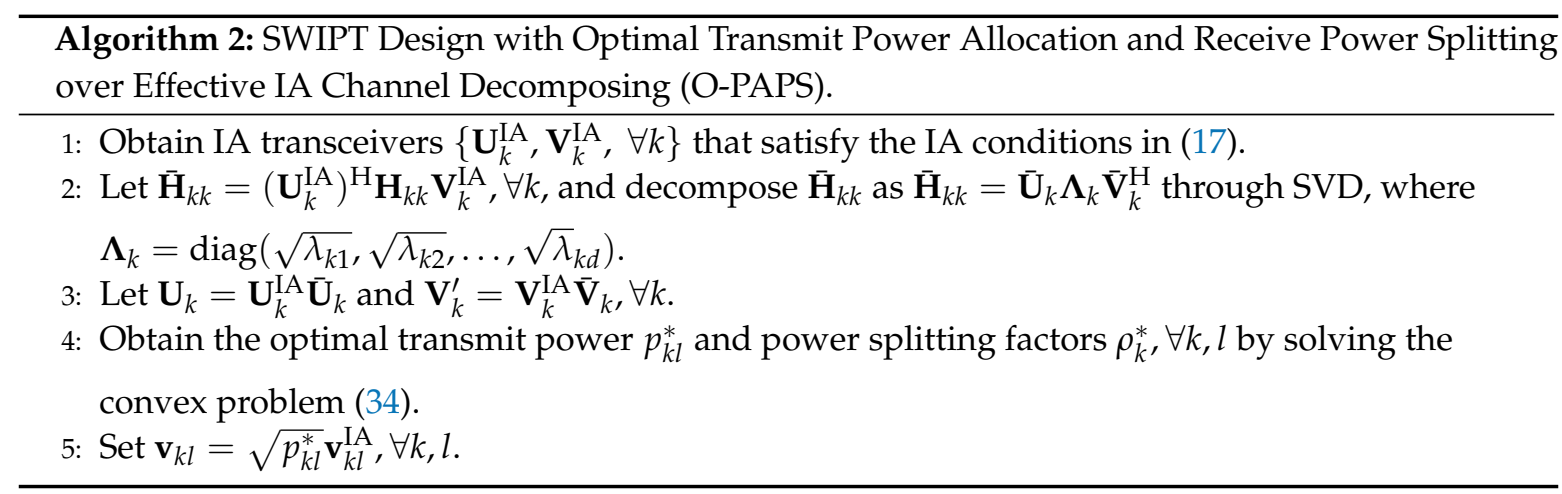

\subsubsection{Closed-Form Power Allocation and Power Splitting Scheme}

Given the IA solution $\left\{\mathbf{U}_{k}^{\mathrm{IA}}, \mathbf{V}_{k}^{\mathrm{IA}}, \forall k\right\}$, we first determine the transmit power to solve the following power minimization problem with only SINR constraints:

$$
\begin{aligned}
\min _{\left\{p_{k l}, \forall k, l\right\}} & \sum_{k=1}^{K} \sum_{l=1}^{d} p_{k l} \\
\text { s.t.: } & \frac{\lambda_{k l} p_{k l}}{\left(\sigma_{n}^{2}+\sigma_{w}^{2}\right)\left\|\mathbf{u}_{k l}\right\|_{2}^{2}} \geq \gamma_{k l}, \forall(k, l) .
\end{aligned}
$$

According to Proposition 1, Problem (35) is always feasible. By further applying Lemma 2, we know Problem (33) is always feasible.

Moreover, Problem (35) can be decoupled over $(k, l)$. Hence, Problem (35) can be decomposed into $\sum_{k=1}^{K} d$ subproblems. For the $l$-th data stream of the $k$-th user, the subproblem is expressed as:

$$
\begin{aligned}
\min _{p_{k l}} & p_{k l} \\
\text { s.t.: } & \frac{\lambda_{k l} p_{k l}}{\left(\sigma_{n}^{2}+\sigma_{w}^{2}\right)\left\|\mathbf{u}_{k l}\right\|_{2}^{2}} \geq \gamma_{k l} .
\end{aligned}
$$

The solution of (36) is then given by:

$$
\hat{p}_{k l}=\frac{\left(\sigma_{n}^{2}+\sigma_{w}^{2}\right)\left\|\mathbf{u}_{k l}\right\|_{2}^{2} \gamma_{k l}}{\lambda_{k l}} .
$$

Following Lemma 1, we next consider optimizing Problem (33) by substitute $p_{k l}$ with $\alpha \hat{p}_{k l}$, where $\alpha \geq 1$ is a scaling factor to be optimized. The optimization problem is then reduced to jointly optimize $\alpha$ and PS factors $\rho_{k}$ under SINR and EH constraints, which is expressed as

$$
\begin{array}{ll} 
& \min _{\alpha,\left\{\rho_{k}, \forall k, l\right\}} \sum_{k=1}^{K} \sum_{l=1}^{d} \alpha \hat{p}_{k l} \\
\text { s.t.: } & \rho_{k} \geq \frac{\sigma_{w}^{2}\left\|\mathbf{u}_{k l}\right\|_{2}^{2} \gamma_{k l}}{\alpha \lambda_{k l} \hat{p}_{k l}-\sigma_{n}^{2} \gamma_{k l}\left\|\mathbf{u}_{k l}\right\|_{2}^{2}}, \\
& 1-\rho_{k} \geq \frac{\psi_{k}}{\xi_{k}\left(\sum_{j=1}^{K} \sum_{m=1}^{d} \alpha \hat{p}_{j m}\left\|\mathbf{H}_{k j} \mathbf{v}_{j m}^{\prime}\right\|_{2}^{2}+n_{\mathrm{r}} \sigma_{n}^{2}\right)}, \\
& 0 \leq \rho_{k} \leq 1, \forall(k, l), \\
& \alpha>1 .
\end{array}
$$


Proposition 3. Given the IA transceivers $\left\{\mathbf{U}_{k}^{I A}, \mathbf{V}_{k}^{I A}, \forall k\right\}$, define $a_{k l}=\sigma_{w}^{2}\left\|\mathbf{u}_{k l}\right\|_{2}^{2} \gamma_{k l}, b_{k l}=\sigma_{n}^{2} \gamma_{k l}\left\|\mathbf{u}_{k l}\right\|_{2}^{2}$ $c_{k l}=\lambda_{k l} \hat{p}_{k l}, f_{k}=\xi_{k} \sum_{j=1}^{K} \sum_{m=1}^{d} \hat{p}_{j m} \xi_{k}\left\|\mathbf{H}_{k j} \mathbf{v}_{j m}^{\prime}\right\|_{2}^{2}$ and $g_{k}=\xi_{k} n_{\mathrm{r}} \sigma_{n}^{2}$. The optimal solution to Problem (38) is given by:

$$
\begin{aligned}
& \alpha^{*}=\max _{\forall(k, l)} \alpha_{k l}^{*}, \\
& \rho_{k}^{*}=\max _{\forall l} \rho_{k l},
\end{aligned}
$$

where $\alpha_{k l}^{*}$ is the solution to the equation $\frac{a_{k l}}{\alpha c_{k l}-b_{k l}}+\frac{\psi_{k}}{\alpha f_{k}+g_{k}}=1(\alpha>1), \rho_{k l}=\frac{a_{k l}}{\alpha^{*} c_{k l}-b_{k l}}$.

Proof. Problem (38) can be expressed as:

$$
\begin{array}{ll} 
& \min _{\alpha,\left\{\rho_{k}\right\}} \alpha \\
\text { s.t.: } & \rho_{k} \geq \frac{a_{k l}}{\alpha c_{k l}-b_{k l}}, \\
& 1-\rho_{k} \geq \frac{\psi_{k}}{\alpha f_{k}+g_{k}}, \\
& 0 \leq \rho_{k} \leq 1, \forall(k, l), \\
& \alpha>1 .
\end{array}
$$

By summing up the SINR constraint with respect to $(k, l)$ and the EH constraint with $k$, Problem (41) can be further reformulated as the following problem:

$$
\begin{array}{ll}
\min _{\alpha} & \alpha \\
\text { s.t.: } & F_{k l}(\alpha) \leq 1, \forall(k, l), \\
& \alpha>1,
\end{array}
$$

where $F_{k l}(\alpha)=\frac{a_{k l}}{\alpha c_{k l}-b_{k l}}+\frac{\psi_{k}}{\alpha f_{k}+g_{k}}$, which monotonically decreases on interval $[1,+\infty)$. According to (37), it is valid that $\frac{a_{k l}}{c_{k l}-b_{k l}}=1$; therefore $F_{k l}(\alpha=1)=1+\frac{\psi_{k}}{f_{k}+g_{k}}>1$. Since $F_{k l}(\alpha)$ monotonically decreases on interval $[1,+\infty)$, the function $F_{k l}(\alpha)=1$ has a unique solution for $\alpha^{*}>1$ on the feasible region of Problem (42). Therefore, we have $\alpha \geq \alpha_{k l}^{*}>1$ to satisfy $F_{k l}(\alpha) \leq 1, \forall(k, l)$. Consequently, the optimal solution of (42) is given by the maximum value among all of the solutions of $F_{k l}(\alpha)=1, \forall(k, l)$, i.e., $\alpha^{*}=\max _{\forall(k, l)} \alpha_{k l}^{*}$, where $\alpha_{k l}^{*}=\frac{B_{k l}+\sqrt{B_{k l}^{2}-4 A_{k l} C_{k l}}}{2 A_{k l}}, A_{k l}=c_{k l} f_{k}, C_{k l}=b_{k l} \psi_{k}-b_{k l} g_{k}-a_{k l} g_{k}$ and $B_{k l}=b_{k l} f_{k}+a_{k l} f_{k}+c_{k l} \psi_{k}-c_{k l} g_{k}$.

After obtaining $\alpha^{*}$, we next discuss how to determine the power split factor $\rho_{k}$. The optimal transmit power scaling factor $\alpha^{*}$ guarantees $F_{k l}\left(\alpha^{*}\right)=\frac{a_{k l}}{\alpha^{*} c_{k l}-b_{k l}}+\frac{\psi_{k}}{\alpha^{*} f_{k}+g_{k}} \leq 1, \forall(k, l)$. Define $\rho_{k l}=\frac{a_{k l}}{\alpha^{*} c_{k l}-b_{k l}}$; it holds that $\frac{\psi_{k}}{\alpha^{*} f_{k}+g_{k}} \leq 1-\rho_{k l}, \forall l$. Therefore, any $\rho_{k l}, \forall l$ can satisfy the EH constraint for the $k$-th user in (41). In order to simultaneously satisfy the SINR constraint in (41), the power splitting factor $\rho_{k}$ can be determined as the maximum value among $\rho_{k l}, \forall l$.

Given $\alpha^{*}$ and $\hat{p}_{k l}$, the transmit precoders are then determined by $\mathbf{v}_{k l}=\sqrt{\alpha^{*} \hat{p}_{k l}} \mathbf{v}_{k l}^{\prime}$. The proposed IA-based SWIPT scheme with the closed-form transmit power allocation and receive power splitting is summarized in Algorithm 3. 


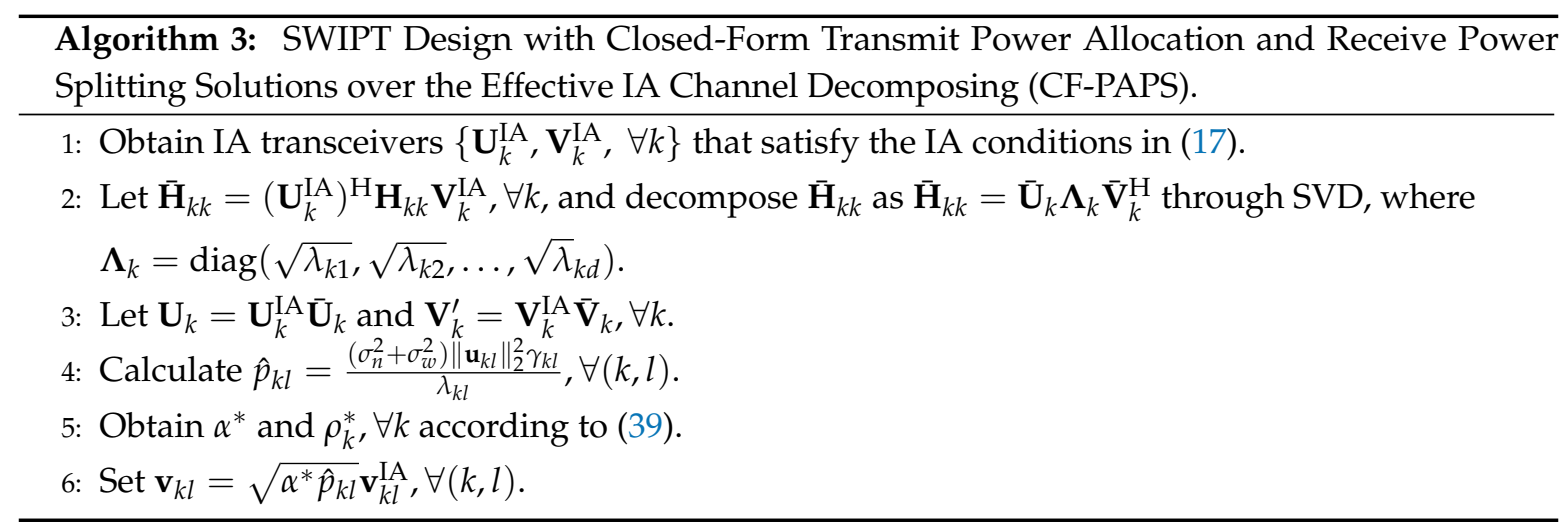

The computational complexity of Algorithm 3 mainly comes from the IA transceiver design process in Step 1. Obviously, different IA algorithms have different computational complexity. For the closed-form solution proposed in [24], the computational complexity mainly depends on the matrix inverting operation. This closed-form solution can be only applied to three-user networks with an equal number of antennas at the transmitters and the receivers, i.e., $n_{t}=n_{r}$, which results in a complexity of $\mathcal{O}\left(n_{t}^{3}\right)$. If the max-SINR or MIL algorithm [30] are applied, the complexity of the IA is about $\mathcal{O}\left(n_{\text {iter }} \max \left(n_{t}, n_{r}\right)^{3}\right)$, considering that the main computations are applied to the SVD and matrix inverting. The complexity of Steps 2-6 depends on the complexity of matrix inversing and SVD, the complexity of which is $\mathcal{O}\left(d^{3}\right)$. Since $d \leq \min \left(n_{t}, n_{r}\right) / 2$, the complexity of Steps $2-6$ can be negligible compared with that of Step 1 . Recall that Step 1 is used for all of the investigated algorithms; the complexity of Algorithm 3 is much lower than that of Algorithm 1 and Algorithm 2. We note that Algorithm 2 and Algorithm 3 can always find feasible solutions if the IA is perfectly achieved. However, for Algorithm 1, there exists a small amount of instances where the feasible solution fails to be obtained.

Note that we have considered the symmetric networks in developing Algorithm 1, Algorithm 2 and Algorithm 3 due to the convenience in derivation and analysis. In fact, the same idea and methods can be generalized into the general asymmetric networks with different numbers of antennas and data streams among users. To achieve this, just replace the $d$ in the formulated design problem (6) and the following related derivationwith $d_{k}, \forall k=1, \ldots, K$.

\section{Simulation Results}

In this section, simulation results are presented to demonstrate the performance of the proposed transceiver design and power splitting schemes in MIMO IC SWIPT systems. We consider the wireless system as described in Section 2 with $K=3$ and $K=4$ users. We assume the entries of $\mathbf{H}_{k j}$ are i.i.d. zero mean complex Gaussian random variables with variance $r_{k j}^{-\beta}$, where $r_{k j}$ is the distance in meters between the $j$-th transmitter to the $k$-th receiver and $\beta$ is the path loss factor. Considering that the SDP-JTDPS scheme may fail to find a feasible solution, we will check the feasibility of the channel realization once it is generated, and we only select the channels that are successfully solved by the SDP-JTDPS scheme. Unless otherwise noted, all of the users are set with the same parameters, i.e., $n_{t}=n_{r}, r_{k l}=r=5, \beta=2.7, \sigma_{n}^{2}=-70 \mathrm{dBm}, \sigma_{w}^{2}=-50 \mathrm{dBm}, \xi_{k l}=\xi=0.8, \gamma_{k l}=\gamma$ and $\psi_{k}=\psi$. The closed-form MIMO linear IA algorithm proposed in [24] is adopted to design IA transceivers for the three-user network, and the MIL algorithm [30] is used for the four-user network. The simulation results are obtained by averaging the simulations of 100 channel realizations. The convex optimization problems in the SDP-JTDPS and O-PAPS schemes are solved using CVX [55] with the SDPT3 solver. All problem instances are simulated on a desktop of Intel(R) Core (TM) i3-2120 CPU at 3.3 GHz and 4 G RAM.

Figure 2 shows the convergence property of the SDP-JTDPS scheme for $\gamma=\{0,20\} \mathrm{dB}$ and $\psi=\{0,10\} \mathrm{dBm}$ for one channel realization of the $(4,4,2)^{3}$ network. It can be observed that the 
convergence speed of the SDP-JTDPS scheme is impacted by the EH threshold. At a low EH threshold (e.g., $\psi=0 \mathrm{dBm}$ ), only several iterations are required to converge. However, when the EH threshold is high (e.g., $\psi=10 \mathrm{dBm}$ ), the convergence speed becomes significantly slow and several hundreds or more need to converge. In general, the higher $\mathrm{EH}$ threshold will require more iterations to converge. Although more iterations are beneficial to the optimized total transmit power, this will result in higher computation complexity. Therefore, a compromise between performance and complexity should be considered for the SDP-JTDPS scheme in practical applications. In the following simulations, unless otherwise specified, we terminate the iteration process once the iteration number reaches 100 or the difference between the successive optimized total transmit powers is less than a predefine threshold, i.e., $10^{-6}$.
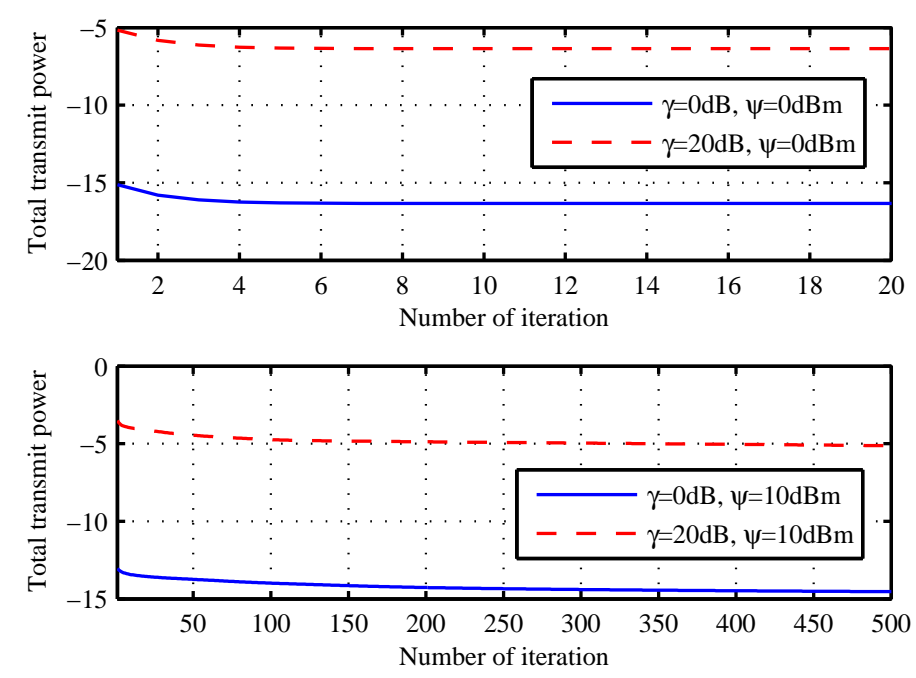

Figure 2. Convergence property of the semidefinite programming (SDP)-joint transceiver design and power splitting (JTDPS) scheme for the $(4,4,2)^{3}$ network.

To gain morn insight into the performance of the proposed SDP-JTDPS scheme, its convergence property is also simulated in a $(6,6,2)^{4}$ network, and the simulation results are given in Figure 3 . It is observed that the convergence trend of the curves in Figure 3 is similar to their counterpart in Figure 2, but having a slower speed. Moreover, it is also observed that the transmit power of the four-user network is lower than the three-user network. This is reasonable, since the four-user network has to be equipped with more transmit antennas than the three-user network in order to satisfy the IA constraints (10) and thus provide more diversity gain for energy harvesting to further lower the transmit power. 

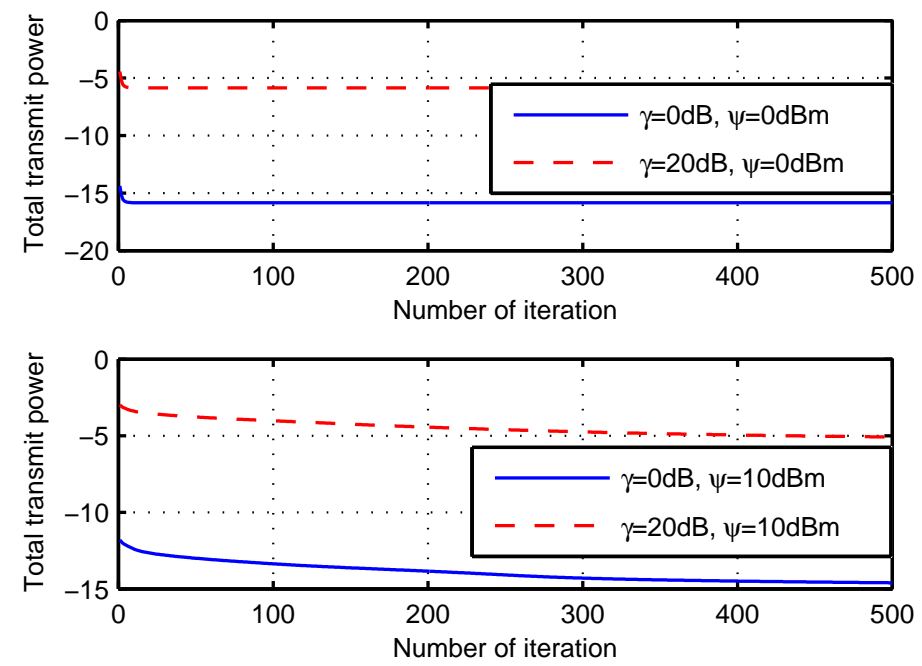

Figure 3. Convergence property of the SDP-JTDPS scheme for the $(6,6,2)^{4}$ network.

Figure 4 shows the empirical cumulative distribution function (CDF) of the output per-stream SINR at the ID receivers given different SINR and EH constraints for the $(4,4,2)^{3}$ network. The SINR target $\gamma$ is set as $20 \mathrm{~dB}$, and the $\mathrm{EH}$ target is set as $0 \mathrm{dBm}$ and $30 \mathrm{dBm}$, respectively. The simulation results are obtained from 100 channel realizations. It can be observed that the SINR targets can always be satisfied by the proposed schemes at any given EH threshold. For the SDP-JTDPS and O-PAPSschemes, the achieved SINR value has the possibility to be higher than the target value. While for the CF-PAPS scheme, the achieved SINR values equal the SINR target with probability of one, which implies that the EH constraints are satisfied with equality by the CF-PAPS scheme.

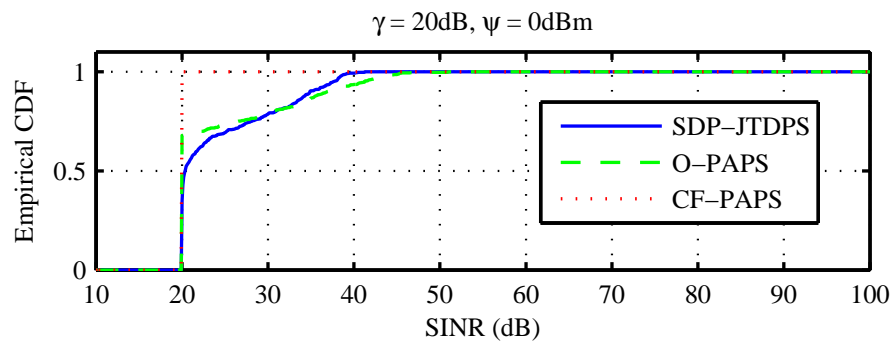

$\gamma=20 \mathrm{~dB}, \psi=30 \mathrm{dBm}$

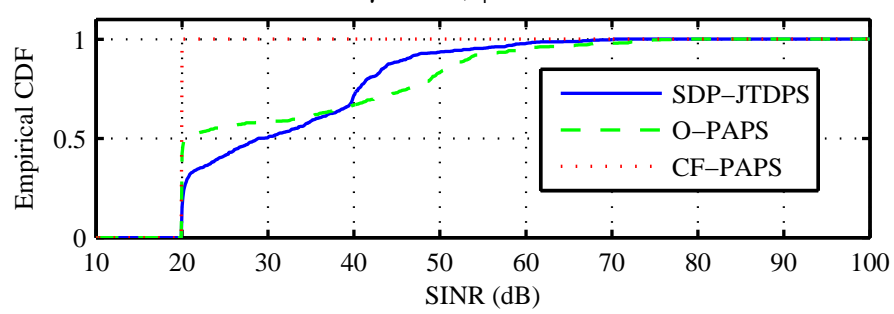

Figure 4. Empirical distribution of achieved SINR at ID receivers with different SINR and energy harvesting $(\mathrm{EH})$ thresholds for the $(4,4,2)^{3}$.

Figure 5 shows the empirical CDF of the per-user harvested energy at the EH receivers given different SINR and EH constraints. The EH target is set as $30 \mathrm{dBm}$, and the SINR target $\gamma$ is set as $0 \mathrm{~dB}$ and $20 \mathrm{~dB}$, respectively. It is observed that all schemes can achieve the $\mathrm{EH}$ targets. In detail, the CF-OPAPS scheme can achieve higher harvested energy than the target value, while the SDP-JTDPS and O-PAPS schemes can achieve the EH target almost perfectly. Therefore, the EH constraints can be satisfied with equality by the SDP-JTDPS and O-PAPS schemes. 

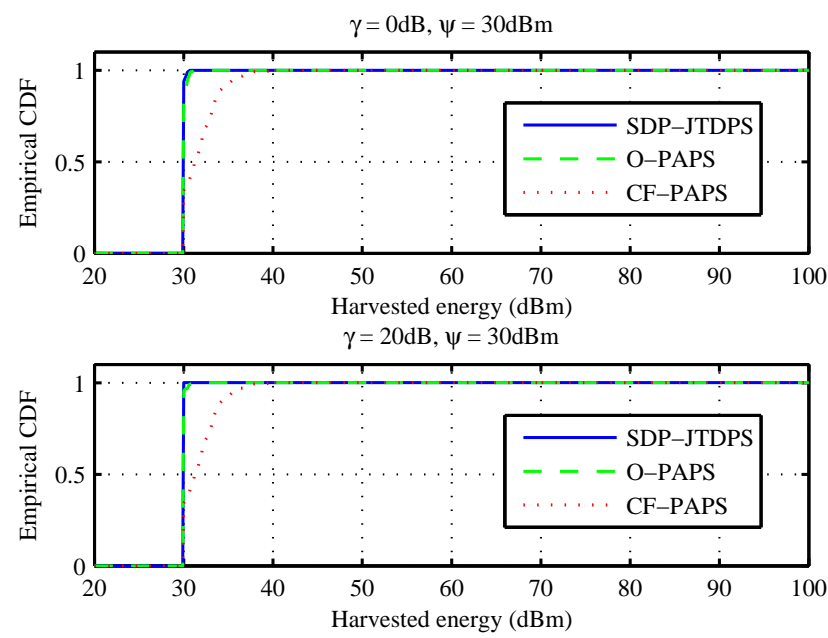

Figure 5. Empirical distribution of harvested energy at ID receivers with different SINR and EH thresholds for the $(4,4,2)^{3}$.

The average total transmit power versus SINR thresholds with the EH threshold $\psi$ fixed is shown in Figure 6. It is observed that more transmit power is needed as the EH threshold increases from $-10 \mathrm{dBm}-30 \mathrm{dBm}$ for any scheme at any given SINR threshold. When the SINR threshold is low, the SDP-JTDPS and O-PAPS schemes achieve almost the same performance, and both of them outperform the CF-PAPS scheme. However, when the SINR threshold is high, the SDP-JTDPS scheme performs worse than the O-PAPS scheme and even worse than the CF-PAPS scheme. Moreover, the gap between the transmit power of the CF-PAPS scheme and the O-PAPS scheme will tend to zero as the SINR threshold becomes high. This implies that the performance of the CF-PAPS scheme is asymptotically the same as that of the O-PAPS scheme and both of them outperform the SDP-JTDPS scheme when the SINR threshold is high.

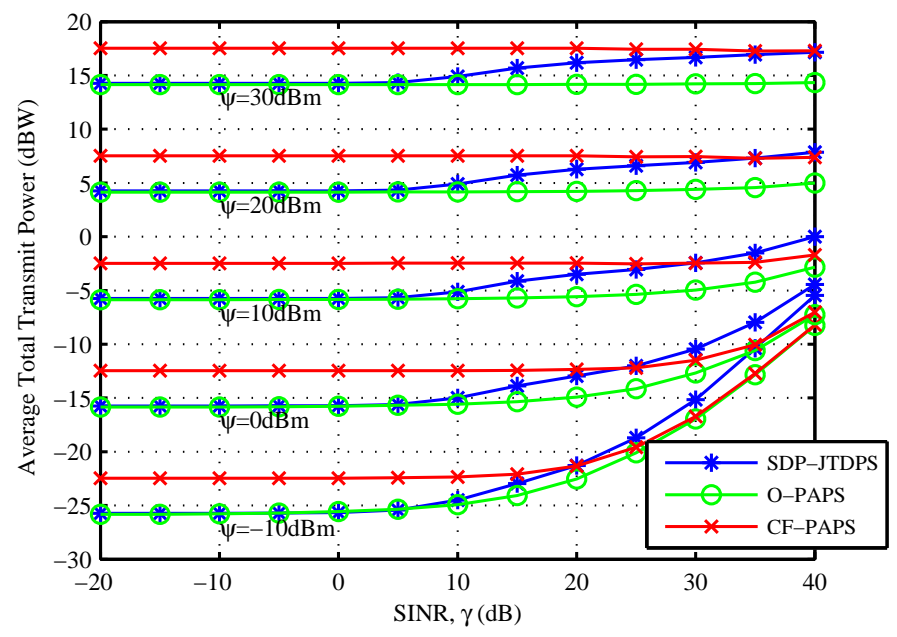

Figure 6. Average total transmit power versus SINR thresholds for the $(4,4,2)^{3}$.

Figure 7 compares the average transmit powers achieved by the three schemes over different EH thresholds with fixed SINR threshold. For the O-PAPS scheme or the CF-PAPS scheme, it can be observed that the average transmit powers for different SINR thresholds will asymptotically tend to be the same when the EH threshold becomes high. In fact, this phenomenon has been shown in Figure 6, where it can be observed that the transmit powers of the O-PAPS scheme or the CF-PAPS scheme are the same for all SINR values at $\psi=30 \mathrm{dBm}$. With $\gamma$ fixed, the higher EH threshold will need more transmit power for any scheme. It is also observed that the SDP-JTDPS scheme and the O-PAPS 
scheme achieve the same performance given the low SINR threshold (e.g., $\gamma=-20 \mathrm{~dB}$ ), but when the SINR threshold becomes high, the SDP-JTDPS scheme performs inferior to the other two schemes significantly at a low EH threshold. It is also observed that at an extremely high EH threshold and a high SINR threshold (e.g., $E H=30 \mathrm{dBm}$ and $\gamma=40 \mathrm{~dB}$ ), the SDP-JTDPS and CF-PAPS schemes' performances are almost the same.

In Figure 8, we compare the proposed algorithms with the DIAscheme proposed in [41] in a network with $M=N=2$ and $d=1$. It is noted that an SDRAOalgorithm has also been proposed in [41], which is a special case of our SDP-JTDPS scheme with $d=1$. That is to say the proposed SDP-JTDPS scheme is equivalent to the SDP-JTDPS scheme if $d=1$. It can be observed that the SDP-JTDPS (i.e., SDRAO) scheme performs the best among all schemes, and the proposed O-PAPS scheme achieves almost the same performance as the DIA scheme. The superiority of the SDP-JTDPS scheme is significant when the SINR threshold is low. However, when the SINR threshold becomes high, the difference between the SDP-JTDPS scheme and that of the DIA or O-PAPS scheme diminishes. Although the proposed O-PAPS scheme performs similar to the DIA on total transmit power, our scheme has the advantage of lower computational complexity, since the SDP solving process is involved in the DIA scheme.

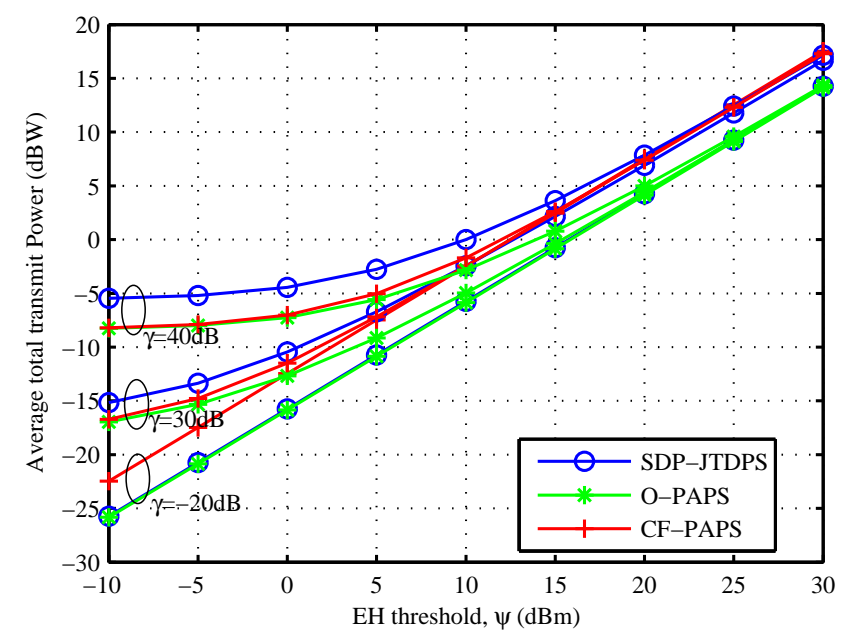

Figure 7. Average total transmit power versus EH thresholds for the $(4,4,2)^{3}$ network.

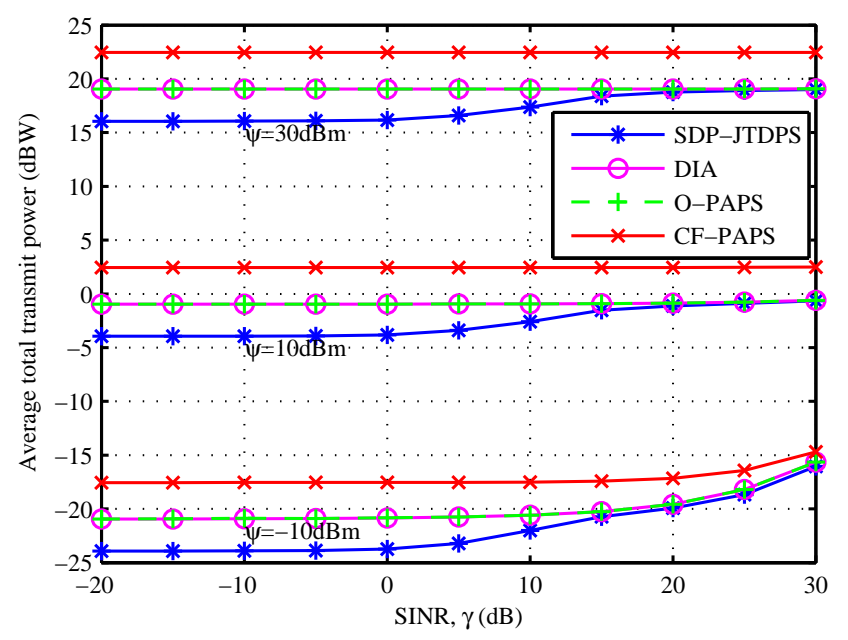

Figure 8. Average total transmit power versus SINR thresholds for the $(2,2,1)^{3}$ network. The schemes proposed in [41] are compared. 
In Figure 9, we compare the proposed algorithms with the DIA scheme proposed in [41] in a $(6,6,2)^{4}$ network over one channel instance. Note that restricted by its design mechanism, only one data stream is utilized in the DIA algorithm in the simulation. It can be observed that the O-PAPS scheme performs the best, and the SDP-JTDPS scheme performs almost the same as the O-PAPS scheme when the SINR threshold is low. This phenomenon is because the SDP-JTDPS scheme has a lower convergence speed when the SINR is high, as shown in Figure 3, while the maximum iteration number is set as 10 in the simulation for saving calculation time. Among all of the schemes, the DIA scheme requires the highest transmit power at any given SINR and EH threshold. This is because only one beamforming vector is utilized at each transmitter in the DIA scheme, while there are two beamforming vectors used in our schemes. Therefore, due to multiplexing gain in our scheme, they achieve better performance in transmit power than the DIA scheme.

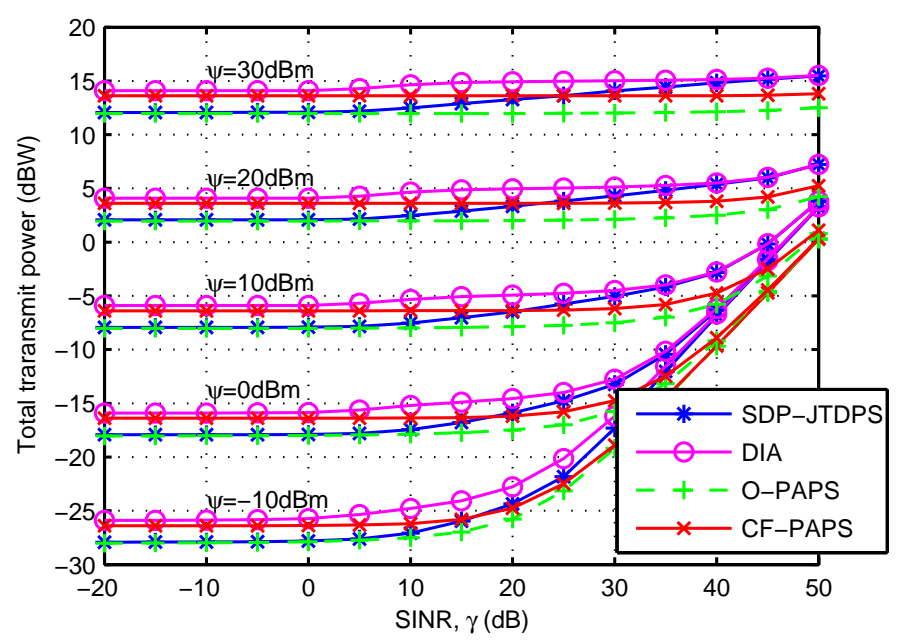

Figure 9. Total transmit power versus SINR thresholds for the $(6,6,2)^{4}$ network over one channel realization. Our schemes are compared with the DIA scheme proposed in [41].

Figure 10 compares the average transmit powers achieved by the proposed schemes over different EH thresholds with a fixed SINR threshold for the $(6,6,2)^{3}$ network. Similar to Figure 7 , the curves of the O-PAPS scheme and the CF-PAPS scheme will asymptotically tend to be the same for a high EH threshold over all given SINR thresholds. The curves of the DIA scheme also asymptotically tend to be the same over all given SINR thresholds. Nevertheless, the curves of the SDP-JTPDS scheme does not tend to be the same over the observed EH scope. This is because again, the convergence speed of the SDP-JTPDS scheme will slow down when SINR becomes high. Considering its high computational complexity, we set the iteration number as 10 in the simulation. If a large enough iteration number is set, we deduce that the curves of the SDP-JTPDS scheme will also asymptotically tend to be the same.

Figure 11 shows the impact of antenna number on the computational complexity of the proposed schemes in terms of average execution time for the $K=3$ network. To facilitate the comparison, the maximum iteration number is set as one for the SDP-JTDPS scheme in the simulations. It is observed that the execution time of all schemes increases with $M$. The computational complexity of the SDP-JTDPS scheme increases nonlinearly with $M$, and the O-PAPS and CF-PAPS schemes increase linearly. The SDP-JTDPS scheme consumes significantly more time than the other two schemes when the antenna number becomes large, e.g., when $M=20$, the average execution time is $69.56 \mathrm{~s}, 1.74 \mathrm{~s}$ and $0.024 \mathrm{~s}$ for the SDP-JTDPS scheme, the O-PAPS scheme and CF-PAPS scheme, respectively. If the maximum iteration number is set to tens or hundreds, the computational complexity of the SDP-JTDPS scheme becomes prohibitive. Therefore, the CF-PAPS and O-PAPS schemes are very attractive for practical applications. 


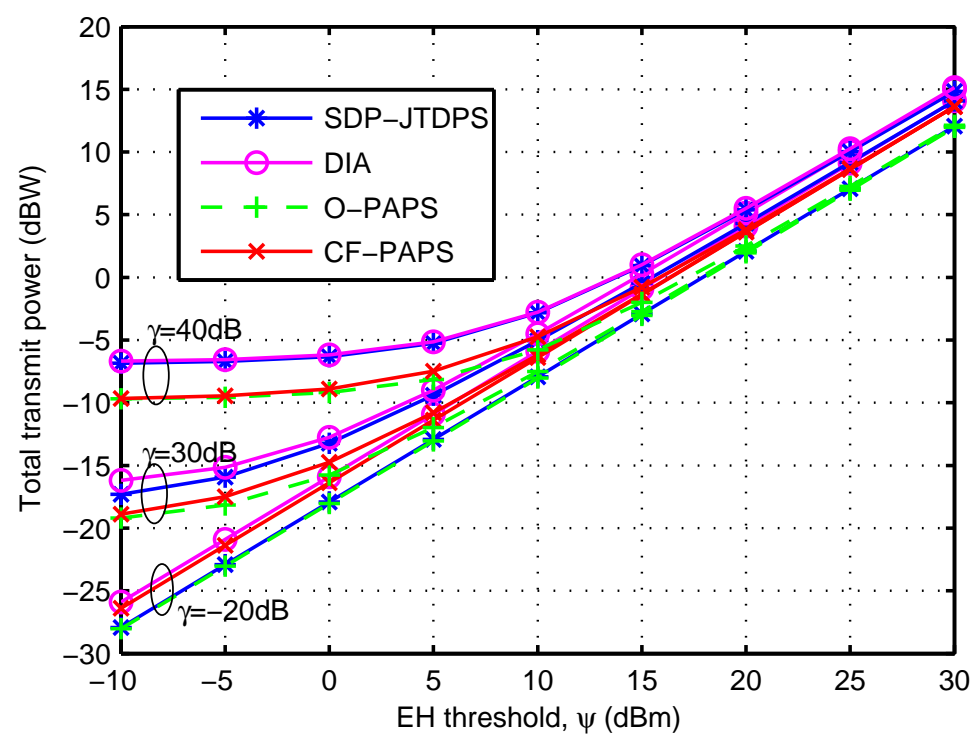

Figure 10. Total transmit power versus EH thresholds for the $(6,6,2)^{4}$ network over one channel realizations. Our schemes are compared with the DIAscheme proposed in [41].

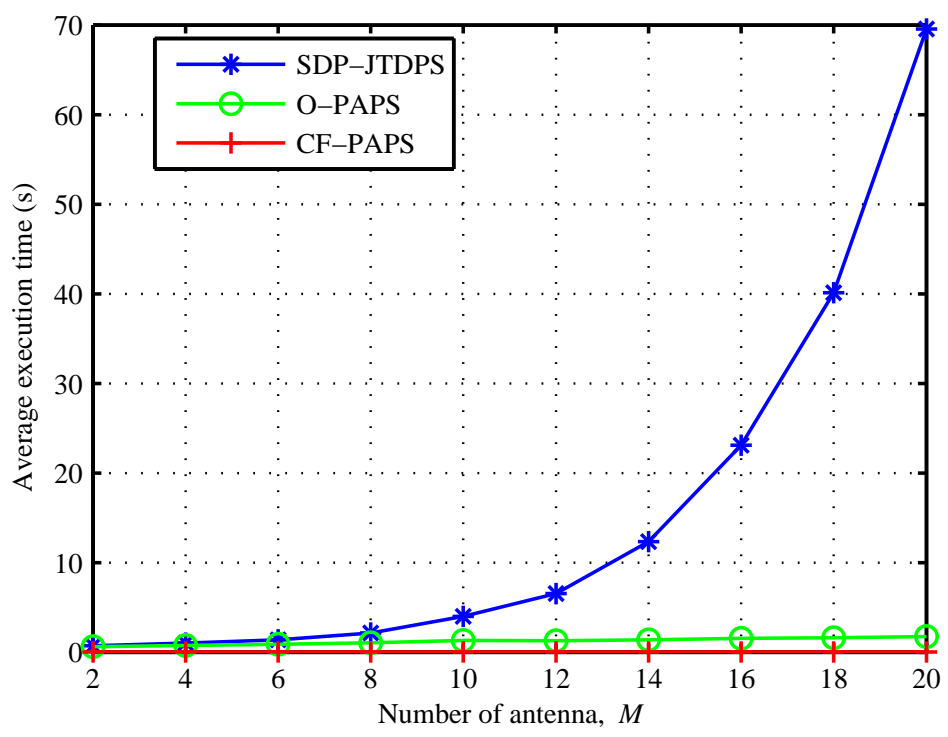

Figure 11. Average execution time versus $M$ at $\gamma=10 \mathrm{~dB}$ and $\psi=10 \mathrm{dBm}$.

\section{Conclusions}

The simultaneous wireless information and power transfer schemes for MIMO interference channel networks have been investigated in this paper. With the aim to minimize transmit power subject to given SINR and energy harvesting constraints, a non-convex joint transceiver design and power splitting problem was formulated. A sufficient condition to guarantee the feasibility of the non-convex problem was derived, which reveals that the problem is always feasible if the system is not interference limited. Based on SDP relaxation, an alternative solving algorithm was proposed by iteratively optimizing the transmitter together with the PS factors and the receiver. The convergence of the proposed framework has been proven. To avoid the high computational complexity of the SDP-based scheme, a low-complexity suboptimal scheme was developed by cascading the traditional IA solutions with a joint transmit power allocation and receive power splitting. Moreover, to further reduce the computational complexity, given the IA solutions, a scheme with a closed-form solution for 
power allocation and PS rations was also derived. Simulation results have shown the effectiveness of the proposed designs in achieving SWIPT.

For future work, the robust designs should be considered for a practical system, where the CSI errors emerge. The CSI error will make the analysis and designs much more complex and, thus, poses challenging problems. Another direction of research is to extend the study to more complicated networks, such as the MIMO IBC networks, the cognitive IA systems, etc.

Acknowledgments: This work was supported by the International S\&T Cooperation Program of China under Grant 2014DFA11640, the National Science Fund of China for Excellent Young Scholars under Grant 61622111, the National Natural Science Foundation of China (NSFC) under Grant 61701269, Grant 61671278, Grant 61601269 and Grant 61371109, the Shandong Provincial Natural Science Foundation of China under Grant ZR2017BF012, the Open Research Fund from Shandong provincial Key Laboratory of Computer Network under Grant SDKLCN-2016-03.

Author Contributions: Anming Dong and Haixia Zhang collaboratively conceived the design schemes, made the theoretical analysis, and wrote the paper. Minglei Shu helped with developing this work in discussion, and helped in simulating and analyzing the results. Dongfeng Yuan gave the guidance of theoretical analysis. All authors have read and approved the final manuscript.

Conflicts of Interest: The authors declare no conflict of interest.

\section{References}

1. Kang, X.; Chia, Y.K.; Ho, C.K.; Sun, S. Cost Minimization for Fading Channels With Energy Harvesting and Conventional Energy. IEEE Trans. Wirel. Commun. 2014, 13, 4586-4598.

2. Ngo, H.Q.; Larsson, E.G.; Marzetta, T.L. Energy and spectral efficiency of very large multiuser MIMO systems. IEEE Trans. Commun. 2013, 61, 1436-1449.

3. Shirazinia, A.; Dey, S.; Ciuonzo, D.; Salvo Rossi, P. Massive MIMO for Decentralized Estimation of a Correlated Source. IEEE Trans. Signal Process. 2016, 64, 2499-2512.

4. Ciuonzo, D.; Salvo Rossi, P.; Dey, S. Massive MIMO channel-aware decision fusion. IEEE Trans. Signal Process. 2015, 63, 604-619.

5. Huang, K.; Zhou, X. Cutting the last wires for mobile communications by microwave power transfer. IEEE Commun. Mag. 2015, 53, 86-93.

6. Prasad, K.N.R.S.V.; Hossain, E.; Bhargava, V.K. Energy Efficiency in Massive MIMO-Based 5G Networks: Opportunities and Challenges. IEEE Wirel. Commun. 2017, 24, 86-94.

7. Varshney, L. Transporting information and energy simultaneously. In Proceedings of the IEEE International Symposium on Information Theory (ISIT), Toronto, ON, Canada, 6-11 July 2008; pp. 1612-1616.

8. Grover, P.; Sahai, A. Shannon meets Tesla: Wireless information and power transfer. In Proceedings of the IEEE International Symposium on Information Theory (ISIT), Austin, TX, USA, 13-18 June 2010; pp. 2363-2367.

9. Krikidis, I.; Timotheou, S.; Nikolaou, S.; Zheng, G.; Ng, D.; Schober, R. Simultaneous wireless information and power transfer in modern communication systems. IEEE Commun. Mag. 2014, 52, 104-110.

10. Liu, L.; Zhang, R.; Chua, K.C. Wireless Information Transfer with Opportunistic Energy Harvesting. IEEE Trans. Wirel. Commun. 2013, 12, 288-300.

11. Zhang, R.; Ho, C.K. MIMO Broadcasting for Simultaneous Wireless Information and Power Transfer. IEEE Trans. Wirel. Commun. 2013, 12, 1989-2001.

12. Xu, J.; Liu, L.; Zhang, R. Multiuser MISO Beamforming for Simultaneous Wireless Information and Power Transfer. IEEE Trans. Signal Process. 2014, 62, 4798-4810.

13. Ikhlef, A. Optimal MIMO Multicast Transceiver Design for Simultaneous Information and Power Transfer. IEEE Commun. Lett. 2014, 18, 2153-2156.

14. Song, C.; Ling, C.; Park, J.; Clerckx, B. MIMO broadcasting for simultaneous wireless information and power transfer: Weighted MMSE approaches. In Proceedings of the Globecom Workshops (GC Wkshps), Austin, TX, USA, 8-12 December 2014; pp. 1151-1156.

15. Shi, Q.; Liu, L.; Xu, W.; Zhang, R. Joint Transmit Beamforming and Receive Power Splitting for MISO SWIPT Systems. IEEE Trans. Wirel. Commun. 2014, 13, 3269-3280. 
16. Wang, F.; Peng, T.; Huang, Y.; Wang, X. Robust Transceiver Optimization for Power-Splitting Based Downlink MISO SWIPT Systems. IEEE Signal Process. Lett. 2015, 22, 1492-1496.

17. Dong, A.; Zhang, H.; Wu, D.; Yuan, D. QoS-constrained transceiver design and power splitting for downlink multiuser MIMO SWIPT systems. In Proceedings of the IEEE International Conference on Communications (ICC), Kuala Lumpue, Malaysia, 23-27 May 2016; doi:10.1109/ICC.2016.7511628.

18. Zhang, H.; Dong, A.; Jin, S.; Yuan, D. Joint Transceiver and Power Splitting Optimization for Multiuser MIMO SWIPT under MSE QoS Constraints. IEEE Trans. Veh. Technol. 2017, 66, 7123-7135.

19. Timotheou, S.; Krikidis, I.; Zheng, G.; Ottersten, B. Beamforming for MISO Interference Channels with QoS and RF Energy Transfer. IEEE Trans. Wirel. Commun. 2014, 13, 2646-2658.

20. Shi, Q.; Xu, W.; Chang, T.H.; Wang, Y.; Song, E. Joint Beamforming and Power Splitting for MISO Interference Channel With SWIPT: An SOCP Relaxation and Decentralized Algorithm. IEEE Trans. Signal Process. 2014, 62, 6194-6208.

21. Zhao, M.M.; Cai, Y.; Shi, Q.; Champagne, B.; Zhao, M.J. Robust Transceiver Design for MISO Interference Channel With Energy Harvesting. IEEE Trans. Signal Process. 2016, 64, 4618-4633.

22. Zhao, N.; Yu, F.; Leung, V. Wireless energy harvesting in interference alignment networks. IEEE Commun. Mag. 2015, 53, 72-78.

23. Zhao, N.; Yu, F.R.; Leung, V.C. Opportunistic communications in interference alignment networks with wireless power transfer. IEEE Wirel. Commun. 2015, 22, 88-95.

24. Cadambe, V.R.; Jafar, S.A. Interference Alignment and Degrees of Freedom of the K-user Interference Channel. IEEE Trans. Inform. Theory 2008, 54, 3425-3441.

25. Maddah-Ali, M.; Motahari, A.; Khandani, A. Communication Over MIMO X Channels: Interference Alignment, Decomposition, and Performance Analysis. IEEE Trans. Inform. Theory 2008, 54, 3457-3470.

26. Birk, Y.; Kol, T. Informed-source coding-on-demand (ISCOD) over broadcast channels. In Proceedings of the Seventeenth Annual Joint Conference of the IEEE Computer and Communications Societies (INFOCOM'98), San Francisco, CA, USA, 29 March-2 April 1998; Volume 3, pp. 1257-1264.

27. Maddah-Ali, M.; Motahari, S.; Khandani, A. Communication Over X Channel: Signalling and Multiplexing Gain University Waterloo, Waterloo, ON; Technical Report; University of Waterloo: Waterloo, ON, Canada, 2006.

28. Weingarten, H.; Shamai, S.; Kramer, G. On the compound MIMO broadcast channel. In Proceedings of Annual Information Theory and Applications Workshop UCSD, San Diego, CA, USA, 12-17 January 2007.

29. Jafar, S.A.; Shamai, S. Degrees of freedom region of the MIMO X channel. IEEE Trans. Inform. Theory 2008, 54, 151-170.

30. Gomadam, K.S.; Cadambe, V.R.; Jafar, S.A. A Distributed Numerical Approach to Interference Alignment and Applications to Wireless Interference Networks. IEEE Trans. Inform. Theory 2011, 57, 3309-3322.

31. Peters, S.W.; Heath, R.W., Jr. Interference alignment via alternating minimization. In Proceedings of the IEEE International Conference on Acoustics, Speech and Signal Processing, Taipei, Taiwan, 19-24 April 2009; pp. 2445-2448.

32. Schmidt, D.A.; Shi, C.; Berry, R.A.; Honig, M.L.; Utschick, W. Minimum mean squared error interference alignment. In Proceedings of the Asilomar Conference Signals, Systems, Computers, Pacific Grove, CA, USA, 29 October-1 November 2009; pp. 1106-1110.

33. Shen, H.; Li, B.; Tao, M.; Wang, X. MSE-based transceiver designs for the MIMO interference channel. IEEE Trans. Wirel. Commun. 2010, 9, 3480-3489.

34. Du, H.; Ratnarajah, T.; Sellathurai, M.; Papadias, C. Reweighted Nuclear Norm Approach for Interference Alignment. IEEE Trans. Commun. 2013, 61, 3754-3765.

35. Sridharan, G.; Yu, W. Beamformer design for interference alignment using reweighted frobenius norm minimization. In Proceedings of the International Workshop on Signal Processing Advances in Wireless Communications (SPAWC), Toronto, ON, Canada, 22-25 June 2014; pp. 469-473.

36. Lee, H.H.; Kim, M.J.; Ko, Y.C. Transceiver design based on interference alignment in MIMO interfering broadcast channels. IEEE Trans. Wirel. Commun. 2014, 13, 6474-6483.

37. Dong, A.; Zhang, H.; Yuan, D.; Zhou, X. Interference Alignment Transceiver Design by Minimizing the Maximum Mean Square Error for MIMO Interfering Broadcast Channel. IEEE Trans. Veh. Technol. 2016, 65, 6024-6037.

38. Perlaza, S.M.; Fawaz, N.; Lasaulce, S.; Debbah, M. From spectrum pooling to space pooling: Opportunistic interference alignment in MIMO cognitive networks. IEEE Trans. Signal Process. 2010, 58, 3728-3741. 
39. Tsinos, C.G.; Berberidis, K. Blind opportunistic interference alignment in MIMO cognitive radio systems. IEEE J. Emerg. Sel. Top. Circuits Syst. 2013, 3, 626-639.

40. Abdulkadir, Y.; Simpson, O.; Nwanekezie, N.; Sun, Y. Space-time opportunistic interference alignment in cognitive radio networks. In Proceedings of the IEEE Wireless Communications and Networking Conference (WCNC), Doha, Qatar, 3-6 April 2016; doi:10.1109/WCNC.2016.7564913.

41. Zong, Z.; Feng, H.; Yu, F.R.; Zhao, N.; Yang, T.; Hu, B. Optimal Transceiver Design for SWIPT in K-User MIMO Interference Channels. IEEE Trans. Wirel. Commun. 2016, 15, 430-445.

42. Zhao, N. Joint Optimization of Power Splitting and Allocation for SWIPT in Interference Alignment Networks. arXiv 2017, arXiv:1701.01952

43. El Ayach, O.; Peters, S.W.; Heath, R.W. The practical challenges of interference alignment. IEEE Wirel. Commun. 2013, 20, 35-42.

44. Yetis, C.M.; Gou, T.; Jafar, S.A.; Kayran, A.H. On feasibility of interference alignment in MIMO interference networks. IEEE Trans. Signal Process. 2010, 58, 4771-4782.

45. Dong, A.; Zhang, H.; Zhou, X.; Yuan, D. On Analytical Achievable Rate for MIMO Linear Interference Alignment with Imperfect CSI. Wirel. Pers. Commun. 2017, 95, 1189-1214.

46. Cadambe, V.R.; Jafar, S.A. Reflections on interference alignment and the degrees of freedom of the K-user interference channel. IEEE Inform. Theory Soc. Newsl. 2009, 59, 5-9.

47. Schubert, M.; Boche, H. Solution of the multiuser downlink beamforming problem with individual SINR constraints. IEEE Trans. Veh. Technol. 2004, 53, 18-28.

48. Negro, F.; Ghauri, I.; Slock, D. On duality in the MISO interference channel. In Proceedings of the Conference Record of the Forty Fourth Asilomar Conference on Signals, Systems and Computers (ASILOMAR), Pacific Grove, CA, USA, 7-10 November 2010; pp. 2104-2108.

49. Chiu, E.; Lau, V.; Huang, H.; Wu, T.; Liu, S. Robust Transceiver Design for K-Pairs Quasi-Static MIMO Interference Channels via Semi-Definite Relaxation. IEEE Trans. Wirel. Commun. 2010, 9, 3762-3769.

50. Tresch, R.; Guillaud, M.; Riegler, E. On the achievability of interference alignment in the K-user constant MIMO interference channel. In Proceedings of the IEEE/SP 15th Workshop on Statistical Signal Processing, Hall Cardiff, UK, 31 August-3 September 2009; pp. 277-280.

51. Liu, S.; Du, Y. A General Closed-Form Solution to Achieve Interference Alignment along Spatial Domain. In Proceedings of the IEEE Global Telecommunications Conference (GLOBECOM), Miami, FL, USA, 6-10 December 2010; doi:10.1109/GLOCOM.2010.5684034.

52. Sung, H.; Park, S.H.; Lee, K.J.; Lee, I. Linear precoder designs for K-user interference channels. IEEE Trans. Wirel. Commun. 2010, 9, 291-301.

53. Wiesel, A.; Eldar, Y.; Shamai, S. Linear precoding via conic optimization for fixed MIMO receivers. IEEE Trans. Signal Process. 2006, 54, 161-176.

54. Luo, Z.Q.; Ma, W.K.; So, A.C.; Ye, Y.; Zhang, S. Semidefinite Relaxation of Quadratic Optimization Problems. IEEE Signal Process. Mag. 2010, 27, 20-34.

55. Grant, M.; Boyd, S. CVX: Matlab Software for Disciplined Convex Programming, Version 2.1. Available online: http:/ / cvxr.com/cvx (accessed on 9 September 2017).

56. Boyd, S.; Vandenberghe, L. Convex Optimization; Cambridge University Press: Cambridge, UK, 2004.

(C) 2017 by the authors. Licensee MDPI, Basel, Switzerland. This article is an open access article distributed under the terms and conditions of the Creative Commons Attribution (CC BY) license (http://creativecommons.org/licenses/by/4.0/). 